\title{
Multi-Winner Spectrum Allocation in Cognitive Radio Networks: A Single-Sided Auction Theoretic Modelling Approach with Sequential Bidding ${ }^{\dagger}$
}

\author{
Monisha Devi $*$;(1), Nityananda Sarma $\ddagger$ and Sanjib K. Deka $\ddagger$ \\ Department of Computer Science and Engineering, Tezpur University, Tezpur, Assam 784028, India; \\ nitya@tezu.ernet.in (N.S.); sdeka@tezu.ernet.in (S.K.D.) \\ * Correspondence: magna@tezu.ernet.in \\ † This paper is an extended version of a conference paper accepted in International Conference on Distributed \\ Computing and Internet Technology (ICDCIT 2020), India. \\ $\ddagger$ These authors contributed equally to this work.
}

check for

updates

Citation: Devi, M.; Sarma, N.; Deka, S.K. Multi-Winner Spectrum Allocation in Cognitive Radio Networks: A Single-Sided Auction Theoretic Modelling Approach with Sequential Bidding. Electronics 2021 10, 602. https://doi.org/10.3390/ electronics10050602

Academic Editor: Francisco Falcone

Received: 26 January 2021

Accepted: 23 February 2021

Published: 5 March 2021

Publisher's Note: MDPI stays neutral with regard to jurisdictional claims in published maps and institutional affiliations.

Copyright: (c) 2021 by the authors. Licensee MDPI, Basel, Switzerland. This article is an open access article distributed under the terms and conditions of the Creative Commons Attribution (CC BY) license (https:/ / creativecommons.org/licenses/by/ $4.0 /)$.

\begin{abstract}
Cognitive radio (CR) has evolved as a novel technology for overcoming the spectrumscarcity problem in wireless communication networks. With its opportunistic behaviour for improving the spectrum-usage efficiency, CR enables the desired secondary users (SUs) to dynamically utilize the idle spectrum owned by primary users. On sensing the spectrum to identify the idle frequency bands, proper spectrum-allocation mechanisms need to be designed to provide an effectual use of the radio resource. In this paper, we propose a single-sided sealed-bid sequential-bidding-based auction framework that extends the channel-reuse property in a spectrum-allocation mechanism to efficiently redistribute the unused channels. Existing auction designs primarily aim at maximizing the auctioneer's revenue, due to which certain CR constraints remain excluded in their models. We address two such constraints, viz. the dynamics in spectrum opportunities and varying availability time of vacant channels, and formulate an allocation problem that maximizes the utilization of the radio spectrum. The auctioneer strategises winner determination based on bids collected from SUs and sequentially leases the unused channels, while restricting the channel assignment to a singlechannel-multi-user allocation. To model the spectrum-sharing mechanism, we initially developed a group-formation algorithm that enables the members of a group to access a common channel. Furthermore, the spectrum-allocation and pricing algorithms are operated under constrained circumstances, which guarantees truthfulness in the model. An analysis of the simulation results and comparison with existing auction models revealed the effectiveness of the proposed approach in assigning the unexploited spectrum.
\end{abstract}

Keywords: cognitive radio; dynamic spectrum access; spectrum allocation; single-sided auction; primary user; secondary user; spectrum opportunities

\section{Introduction}

The regulation of radio spectrum usage is guided by a conventional procedure called static spectrum assignment that permits licensed users to access portions of the spectrum on a long-term basis. However, reports from the Federal Communications Commission (FCC) [1], an independent agency of the United States, reveal that a significant section of the licensed spectrum remains underutilized by its legitimate owners, both spatially and temporally. Such occurrences create spectrum opportunities (or spectrum holes) in the network. On the contrary, recent advances in wireless and mobile technologies have led to insufficiency of the radio spectrum, creating new challenges in resource management. Therefore, with an intention to dynamically exploit the unused spectrum of licensed users or primary users (PUs), cognitive radio (CR) [2-4] presents itself as a promising technology that uses dynamic spectrum access (DSA) [5] techniques and enables the opportunistic 
use of the spectrum holes by unlicensed users or secondary users (SUs) while preventing any destructive interference with the PUs. To facilitate dynamic spectrum allocation, one of the key elements in CR networks is spectrum sensing, during which SUs can discover their spectrum opportunities over a wide range of frequency bands. Once the free channels are known amongst the SUs, there arises a need to design a proper spectrum-allocation mechanism so that unused channels can be fairly distributed according to the demand of the SUs. Although spectrum allocation has been widely explored by several researchers in recent years, unfortunately, their work has ignored certain CR constraints that need to be considered in order to achieve allocation patterns with enhanced spectrum utilization. Different allocation models [6,7] have been developed for CR networks (CRNs). Amongst them, auctions are well-known market-based mechanisms that can efficiently manage the scarce radio resource. Several auction-based models have been designed for CRNs, but none of these approaches incorporate the network constraints that appear in CRNs. As such, there can be situations where these models result in a degraded performance in terms of spectrum utilization.

An auction $[8,9]$ is the process of selling goods to relevant bidders with the return of some monetary profit. Conventional single-sided auctions are being conducted worldwide by government bodies to assign the spectrum to the wireless service providers (WSPs) who submit the highest bid values [10]. However, an auction designed for a CR network differs from such conventional auctions. The prime intention of conventional auctions is to earn high income in exchange for the leased spectrum. However, auction theoretic models for CRNs are designed to boost the spectrum utilization, without concentrating much on the auctioneer's revenue. Both single-sided auctions and double-sided auctions are applicable in a CR environment. In a single-sided auction, the primary owner (primary base station) of a network takes up the role of auctioneer and earns a monetary profit. The primary owner (PO) leases the channels left vacant by the PUs in the network. These available channels, being the auctioned items, get bid for by the SUs, who act as bidders. However, when the POs of the network take part in the auction as sellers and they compete amongst themselves by submitting ask values for their channels, this formulates a double-sided auction. A third party acts as the auctioneer who decides the clearing price based on the bid values and ask values received from the SUs and POs, respectively. However, a PO in a double auction can sell its channel only when its ask value is less than the winning bid value. Otherwise, the channel from the PO remains unassigned.

In this paper, we focus on a single-sided auction mechanism that is being deployed for spectrum allocation in CRNs, as shown in Figure 1. We consider a sealed-bid auction where bidders (SUs) privately communicate their bid values to the auctioneer; no bidder can realize the bid values of another bidder in the game. As such, there appears no information exchange among the SUs, which thereby reduces the communication overhead. Additionally, an auction formulation designs the model in such a way that every participant gets an equal opportunity to win the game, and bidders submitting higher valuations have a greater chance of picking up the auctioned item. In today's scenario, CR technology can be integrated with the $5 \mathrm{G}$ network, where the flexibility in spectrum utility provided by CR can help to achieve higher capacity in such networks [11,12]. Including auctions in designing radio spectrum allocation can impart better coordination with PUs while availing the dynamic spectrum in 5G networks, since PUs themselves are willing to lease their spectrum. Additionally, operators in such a network may not want to communicate due to security reasons. Here, auction mechanisms are worthwhile since operators need to convey their bids only to the auctioneer. Similarly, IoT-based applications $[13,14]$ can also be addressed with CR. With the heavily increasing demand of IoT objects, it can be seen that in the near future, the allocation of the spectrum to these objects will become inadequate. In such an environment, spectrum-allocation mechanisms in CR could help the IoT objects to intelligently access the unused spectrum. As such, $\mathrm{CR}$ would provide a helping hand to different IoT-based healthcare applications, environmental applications, in-home applications, etc. 


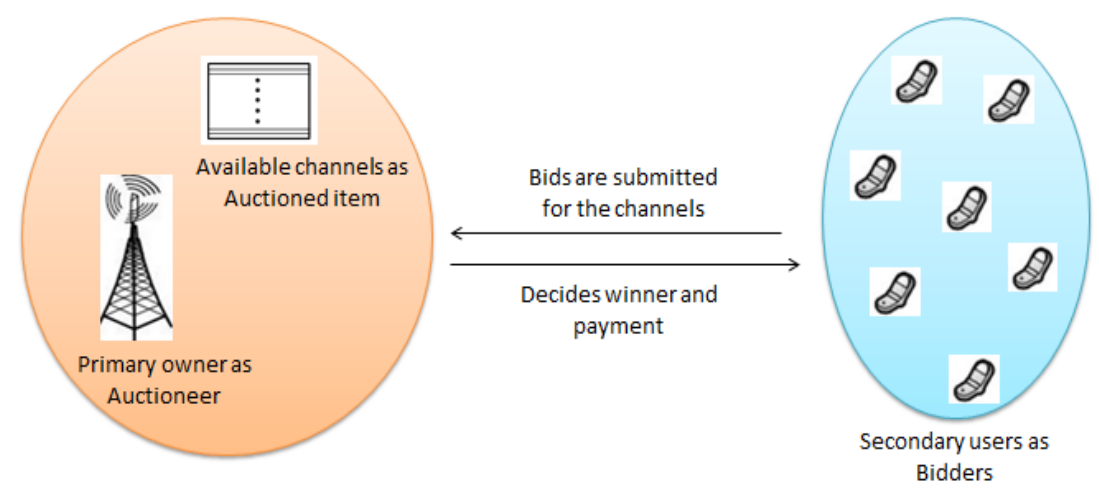

Figure 1. Single-sided auction in cognitive radio networks.

\subsection{Motivation}

Most of the existing auction-based approaches for CRNs deploy a double-sided auction that primarily concentrates on earning a good revenue for the sold channels. However, when a double auction is implemented, based on its allocation condition, there can be some channels that are left unassigned even when there are SUs who are looking for the underutilized channels. Such a condition restricts the use of the radio spectrum. Thus, to enhance the spectrum utilization, a single-sided auction becomes favourable, where all the channels can be allocated while abiding by the network constraints. Additionally, the strategy of monetary bid submission may not be suitable for formulating the spectrumallocation problem in a CRN. When monetary amounts are used for bidding, it is not always necessary that the bidder with the highest bid value who wins the auction makes the best use of the assigned channel for its transmission. Instead, if bids are submitted in terms of a particular channel characteristic, such as the bandwidth, data rate, etc., then we can achieve a better usage of the spectrum bands. Apart from these points, there are certain network challenges that need to be addressed while allocating a channel in a CRN. One such challenge is the variation in the spectrum opportunities (SOPs) of the SUs. The dynamics in SOPs may not allow every SU to sense all the free channels as available. Accordingly, before bidding for the channels, the channel set available at each SU should be studied so that every winning SU receives a channel that is actually available at the SU. Another challenging issue in CRNs is related to the channel-availability time. If a PU wants to reclaim its channel that is currently being used by an SU for its transmission, then the SU immediately vacates the channel, which in turn disturbs the transmission of the SU. Since only one channel will be assigned to an SU in this model, there arises the need to look for the availability time of the channels offered for auction so that an SU can look for only those channels whose availability times will be longer than the time duration for which the SU needs the channels. Otherwise, if an SU wins a channel and, while it is using the channel, the SU's transmission gets interrupted due to the PU activity, then this wastes the spectrum given to that particular SU, resulting in reduced spectrum utilization. Prior work on auction-based models in CRNs have not covered the issues related to the dynamics in SOPs and varying channel-availability time, due to which spectrum utilization as well as network throughput becomes affected. Hence, motivated by these observations, we planned to develop an efficient allocation mechanism for CRNs that could address the challenges that we have explored.

In this paper, we propose a sequential auction-based multi-winner (SAMW) spectrumallocation framework where the PO serves as the auctioneer and decides a noncooperative auction game among SUs (bidders). It extends the work carried out in [15], which discusses an allocation mechanism that applies a concurrent bidding policy to auction heterogeneous channels. The authors in [15] proposed a multi-winner auction mechanism where channels differ with respect to their maximum allowable transmission power, and to achieve a better performance in spectrum utilization, all the available channels are auctioned simultaneously so that each SU can choose its most preferred channel. In this work, we consider 
that the channels offered for auction are homogeneous, due to which sequential bidding becomes favourable for bid submission. To perform the spectrum-sensing process, we assume that the sensing result of every SU is available to us through the basic energy detection method [16]. Moreover, with the radio spectrum being interference limited and reusable among geographically separated users, this work enables multiple noninterfering bidders to share a single idle channel at a time. Such a condition facilitates an increase in spectrum utilization along with the revenue earned by the auctioneer. Furthermore, the proposed model ensures truthfulness for preventing any kind of manipulation of the bid values. This makes every SU bid a true valuation, while the SU is completely unaware of any other bidder's bidding strategy. To evaluate the performance of our proposed model, we conducted simulation experiments in the MATLAB platform. The experimental results for our model are compared with some existing work to analyse the performance improvement.

\subsection{Contribution}

This paper makes the following key contributions:

- With the channels being homogeneous, we apply the sequential bidding strategy for collecting the bids since the bid values from SUs can be improved in each round, which will in turn improve the spectrum utilization. The process of sequential bidding allows every channel to be auctioned one after another, and once an SU obtains its access to a channel, the SU-channel pair cannot further participate in the allocation process.

- Bids from an SU are decided in terms of the rate of data transfer that can be applied over the auctioned channel. There can be different bid values from an SU for each auctioned channel, even though the channels are homogeneous. This is because the bid values from an SU are determined while studying the dynamics in the SOPs of the $\mathrm{SU}$ and the availability time of the auctioned channels. Existing auction models with homogeneous channels allow an SU to submit a uniform bid value for every channel that is auctioned. Such an assumption ignores the network constraints that can appear in a CRN.

- A sealed-bid single-sided auction framework is adopted to address the spectrumallocation problem, where the channel distribution is restricted to a single-channelmulti-user allocation constraint, which declares that each user (SU) can obtain, at most, one channel at a time, but a channel can be assigned to multiple non-interfering users at the same time.

- We developed two algorithms, a group-formation algorithm and spectrum-allocation algorithm, to plan the winner-determination strategy. Then, we developed a pricing algorithm that charges the winning bidders in the return of their respective assigned channels.

\subsection{Organization}

The rest of the paper is organized as follows. Section 2 reviews the related work. The proposed spectrum-auction model is elaborately discussed in Section 3, where the subsections refer to the system model, the challenges that can arise in CRNs, the proposed auction mechanism, and proof of the auction properties, respectively. Section 4 provides the performance evaluation with a discussion of the simulation results. Finally, Section 5 provides the conclusion and future directions.

\section{Related Work}

Many spectrum-sharing approaches deploying different allocation models, such as graph theory [17,18], game theory [19], evolutionary theory [20], and so forth, have been proposed for CRNs. Apart from these, auction theory provides a different perspective for spectrum allocation where PUs themselves lease their unused channels temporarily to the desired SUs. The authors in [21] formulated a bandwidth auction for both single-PU and multi-PU scenarios. A dynamic-updating algorithm plans the spectrum- 
assignment pattern, which can be achieved with the Nash equilibrium. Kash et al., in [22], define bucketing and ironing techniques for developing a scalable strategy-proof auction mechanism for spectrum sharing amongst shared and exclusive-use bidders. A bipartite graph-based auction model was developed in [23] where SUs and channels form the two vertex sets. Finding a maximal weight matching in the bipartite graph defines the winnerdetermination strategy to assign one channel to only one SU. Then, to obtain the revenue of the auctioneer, the authors used the general Vickrey Clarke Groves (VCG) auction. In [24], Amraoui et al. designed another single-sided auction scenario with a first-price auction and English auction. The first-price auction picks the highest bidding user for channel allocation. However, in the English auction, multiple rounds are executed to obtain the winning bidder. Two online-auction models for sharing randomly available spectrum are discussed in [25]. Gao et al., in [26], discuss the problem of maximizing the primary owner's (auctioneer) revenue. Channels are characterised by their channel capacity, and for the bidding process, quality-price combinations are taken up such that a feasible contract can be achieved to provide a fair channel allocation. Another multi-item-based auction mechanism is proposed in [27] that allows every SU to obtain a single channel at a time. The algorithm developed to share the spectrum uses the first-price auction model, allowing the highest-paid bidder to win. A bandwidth or power auction is discussed in [28], where homogeneous resources are sequentially auctioned by following a second-price auction. The authors in [29] focus on revenue generation for the auctioneer by using the concept of virtual valuation during bid submission, and a suboptimal solution was defined to formulate the revenue-maximization problem. Another single-channel-allocation-based auction model is discussed in [30], where the channel-availability time is taken into consideration for deciding the bid value, and adjacent channels get assigned to non-interfering SUs such that each SU obtains a single channel at a time. Similarly, VERITAS, in [31], was designed as a truthful single-sided buyer-only auction to manage the dynamic spectrum market, which further aims at improving the spectrum utilization. Several double-auction models have also been designed to resolve the channel-allocation problem. A double-auction mechanism that models spectrum reuse as an SINR model was deployed in [32]. This approach primarily aims at optimizing the profit and energy across the network and allows every SU to be assigned only one channel. In [33], PreDA uses the concept of the preference list (based on SINR values) to gather bids from the SUs. The formation of virtual groups encourages channel reuse in the model along with multi-channel allocation. Another double-auction model was proposed in [34], where both the seller and buyer sides are decoupled to organize the spectrum separately. On the buyer side, graph partitioning and the further merging of the subgraphs determines the allocation pattern. Consecutively, the sellers follow a traditional method to decide the winners and their respective revenue. In the auction model developed in [35], both the PU and the SUs are allowed to share a common channel constrained to a threshold limit of the interference temperature. TAMES, in [36], involves a multi-seller-multi-buyer double auction that creates spatial reusability via an interference graph with sequential grouping. In [37], another online-auction model named LOTUS was designed that introduces the concept of the interference discount. An adaptive-auction mechanism is discussed in [38], where two different auctions, a local auction and global auction, are implemented with an intention to improve the revenue of the sellers. In [39], an auction is carried out in a cooperative CRN to resolve both spectrum allocation and relay allocation by proposing two different auction schemes. Another relay-based auction model is introduced in [40], which performs relay selection using a Vickrey auction and uses both decode-and-forward and amplify-and-forward protocols for sharing the spectrum. Table 1 compares the existing research that uses auctions for spectrum allocation in CRNs. 
Table 1. Literature study on auction models used for spectrum allocation in cognitive radio networks (CRNs).

\begin{tabular}{cccc}
\hline Reference & Spectrum Reuse & $\begin{array}{c}\text { Dynamics in } \\
\text { SOPs }\end{array}$ & $\begin{array}{c}\text { Channel Availability } \\
\text { Time }\end{array}$ \\
\hline$[21]$ & $\times$ & $\times$ & $\times$ \\
{$[22]$} & $\checkmark$ & $\times$ & $\times$ \\
{$[23]$} & $\times$ & $\times$ & $\times$ \\
{$[24]$} & $\checkmark$ & $\times$ & $\times$ \\
{$[26]$} & $\checkmark$ & $\times$ & $\times$ \\
{$[27]$} & $\times$ & $\times$ & $\times$ \\
{$[28]$} & $\times$ & $\times$ & $\times$ \\
{$[29]$} & $\checkmark$ & $\times$ & $\times$ \\
{$[31]$} & $\checkmark$ & $\times$ & $\times$ \\
{$[32]$} & $\checkmark$ & $\times$ & $\times$ \\
{$[33]$} & $\checkmark$ & $\checkmark$ & $\times$ \\
{$[34]$} & $\checkmark$ & $\times$ & $\times$ \\
{$[35]$} & $\checkmark$ & $\times$ & $\times$ \\
{$[36]$} & $\checkmark$ & $\checkmark$ & $\times$ \\
[37] & $\checkmark$ & $\times$ & $\times$ \\
\hline P39] & $\checkmark$ & $\times$ & $\times$ \\
\end{tabular}

A literature survey on auction designs unveiled that single-sided auctions have not been extensively studied in CRNs. In a practical scenario, a cellular network conducts the single-sided auction, where a base station acts as the auctioneer. When the idle channels of the users are auctioned among the SUs (of an ad hoc CRN), the spectrum utilization can be significantly improved with the single-sided auction, and revenue is drawn by the network provider. Additionally, the survey shows that the existing approaches have not incorporated certain network constraints of CRNs, which need to be investigated in further research work. The dynamics in the SOPs and differences in the availability time of the unused channels are two such network constraints, which when integrated in the auction model, can provide a better spectrum usage as well as being able to increase the network throughput. This study developed an auction mechanism with sequential bidding that permits channel reusability while taking into account both the network constraints.

\section{Auction Model for Spectrum Allocation in CRN}

This section provides an elaborate discussion of our proposed model. Additionally, challenges that appear in CRNs and proofs of auction properties are discussed in this section.

\subsection{System Model}

We assume there is a network with both primary and secondary users. Primary users allow opportunistic access to their free channels to secondary users through a singlesided auction model. True valuations are submitted as bid values while abiding by the availability time constraint of the channels. Winner determination and payment strategies are carried out by the auctioneer with an aim to improve the overall spectrum utilization. Spectrum allocation applies the channel-availability constraint among the SUs and singlechannel-multi-user-allocation constraint for spectrum reuse to achieve an efficient allocation mechanism.

A cognitive radio network consisting of $N$ SUs (bidders), $\mathcal{N}=\{1,2,3, \ldots, N\}$, coexists with a primary network. PUs are willing to give away a total of $M$ idle channels, $\mathcal{M}=\{1,2,3, \ldots, M\}$, among $N$ SUs, where it is assumed that $N>M$. The PO auctions the channels left unused by PUs while allowing them to be reused by non-interfering users and, in return, earns a revenue. To communicate with the auctioneer, a dedicated commoncontrol channel (CCC) in the licensed band [41] can be utilized amongst the SUs along 
with the OFDMA access mechanism. Now, to discover the channel availability for each SU, spectrum sensing needs to be carried out before triggering the allocation process, where the same set of channels may not be available to every SU. Such a variation in the SOPs of the SUs arises due to differences in SU capabilities [42]. This constructs a channel-availability matrix, $C=\left\{c_{i j} \mid c_{i j} \in\{0,1\}\right\}_{N \times M}$, where $c_{i j}=1$ if SU $i$ senses channel $j$ and finds it to be available; otherwise, $c_{i j}=0$. According to the channel-availability constraint, a channel $j$ can be assigned to an $\mathrm{SU} i$ only if $c_{i j}=1$. The interference relationship among geographically separated SUs is represented using an interference matrix, $X=\left\{x_{i k} \mid x_{i k} \in\{0,1\}\right\}_{N \times N}$, where $x_{i k}=1$ if SUs $i$ and $k$ cannot be assigned the same channel simultaneously since they are interfering; otherwise, $x_{i k}=0$. Moreover, the SUs in this model look for channels with higher channel-availability time, since due to particular PU activity, a free channel may become reclaimed by the licensed user, which seems to disturb the data transmission of the SU. The availability time of a channel can be estimated during the sensing phase accordingly, as in [43]. In [43], every channel is configured as an ON-OFF model where the OFF phase specifies the time period in which the owner leaves the channel unused. It is during this period when the SUs can utilize the channel without causing any interference to the owner. Thus, with $T_{A(j)}$ being the channel-availability time for a channel $j$, SU $i$ submits its bid for channel $j$ only when the channel requirement time of $S U i$ for channel $j, T_{R(i j)}$, is less than or equal to $T_{A(j)}$. For every SU who senses channel $j$ as available, $T_{A(j)}$ is approximately similar. In this model, we assume that the transmission time and propagation delay together give the channel requirement time, $T_{R(i j)}$. The distance to the receiver and propagation speed provide the propagation delay for the SU $i$. To obtain the transmission time, the size of the message transmitted by the SU $i$ and data rate over channel $j$ need to be used. As such, on auctioning a channel, the bid value determined by an SU for the channel is the data rate that the SU uses for its transmission over the channel. Another consideration is for homogeneous channels, where all the available channels are similar in their characteristics. In the literature, when channels are homogeneous, authors consider that an SU chooses a uniform valuation of all the channels. However, in this model, the bid submitted by an $S U i$ for each channel $j$ differs with respect to $T_{A(j)}$. Now, on auctioning a channel $j$, the $\mathrm{SU} i$ decides the valuation for the channel, given as $v_{j i}$. Initially, when channel $j$ is auctioned, the $T_{R(i j)}$ of $\mathrm{SU} i$ is obtained by taking the channel capacity of $j$ (the channel capacity is same for all the channels). Accordingly, $v_{j i}$ is decided as per the following conditions.

- If $c_{i j}=0$, then $v_{j i}=0$, since the channel is unavailable at the SU.

- If $c_{i j}=1$ and $T_{R(i j)}>T_{A(j)}$, then $v_{j i}=0$. With $v_{j i}<$ the channel capacity, $T_{R(i j)}$ will increase and cannot proceed towards $T_{A(j)}$.

- If $c_{i j}=1$ and $T_{R(i j)}=T_{A(j)}$, then $v_{j i}=$ the channel capacity. With $v_{j i}<$ the channel capacity, $T_{R(i j)}>T_{A(j)}$.

- If $c_{i j}=1$ and $T_{R(i j)}<T_{A(j)}$, then $v_{j i}<$ the channel capacity, but $v_{j i}$ is chosen such that the condition $T_{A(j)} \geq T_{R(i j)}$ continues to hold.

For instance, consider a channel $C 1$ with a channel capacity of 4.2 bps and $T_{A(1)}$ of $2.806 \mathrm{~s}$. Three SUs, S1, S2, and S3, have message sizes of 10,8 , and 12 bits and propagation delays of $0.30,0.20$, and $0.45 \mathrm{~s}$. Thus, $v_{11}=4.07(10 / 4.07+0.30=2.757<2.806), v_{12}=3.50$ $(8 / 3.50+0.20=2.485<2.806)$, and $v_{13}=0(12 / 4.20+0.45=3.30>2.806)$. Therefore, for $S 3$, the condition cannot be satisfied even on submitting 4.2 as the bid. Moreover, $v_{j i}$ is decided only when $c_{i j}=1$. To represent the channel assignments among SUs, a channel-allocation matrix $A=\left\{a_{i j} \mid a_{i j} \in\{0,1\}\right\}_{N \times M}$ is used, where $a_{i j}=1$ if SU $i$ gets access to channel $j$; otherwise, $a_{i j}=0$. With the allocation constraint stating that one SU can acquire, at mos, $t$ one vacant channel at a time, the rows of the allocation matrix become imposed on $\sum_{j=1}^{M} a_{i j} \leq 1, \forall i \in \mathcal{N}$. Now, the SUs look to submit their bid values for the valuated channel wherein $b_{j i}$ represents the bid given away by $\mathrm{SU} i$ to get access to channel $j$. Every SU, constrained to the sequential-bidding condition, submits the valuation as its bid value, which assures a truthful auction. As such, when channel $j$ is auctioned among SUs, a bidding 
vector $B_{j}$ is built up such that $B_{j}=\left\{b_{j 1}, b_{j 2}, \ldots, b_{j i}, \ldots, b_{j N}\right\}$, where bid $b_{j i}$ is given by Equation (1).

$$
b_{j i}= \begin{cases}v_{j i} & \text { if } \sum_{q=1}^{M} a_{i q}=0 \\ 0 & \text { otherwise }\end{cases}
$$

Therefore, for $M$ channels, the bidding vectors formed during the sequential strategy are $B_{1}, B_{2}, \ldots, B_{M}$. Incorporating a sealed-bid auction game in the model takes bid values as private information from the bidders. Thereafter, once a channel is allocated to the winner SUs, every winner SU $i$ has to pay a price, $p_{i}$, to the auctioneer. $P=\left\{p_{1}, p_{2}, \ldots, p_{i}, \ldots, p_{N}\right\}$ represents a price vector where $p_{i}$ is the expense incurred by $\mathrm{SU} i$ on acquiring a particular channel. However, during the implementation of the pricing strategy, situations may arise where the PO may receive zero payment from a winner SU. To enable the PO to earn a minimal payment for its leased channels, we consider that the PO sets a reserve price on auctioning each channel sequentially. $r_{j}$ represents the reserve price for channel $j$ when the channel is auctioned in the $j$ th round, and $r_{j}$ can be obtained using Equation (2).

$$
r_{j} \leq \min \left\{b_{j i} \in B_{j}\right\} \quad \text { s.t. } b_{j i} \neq 0, r_{j} \neq 0
$$

Thus, when channel $j$ gets assigned, the PO earns at least the reserve price $r_{j}$ as its revenue. The utility earned by an $\mathrm{SU}$ is the difference between the valuation of the user for a channel and the price paid on acquiring the channel. The utility, $u_{i}$, of an SU $i$ for a channel $j$ can be represented as shown in Equation (3).

$$
u_{i}= \begin{cases}v_{j i}-p_{i} & \text { if } a_{i j}=1 \\ 0 & \text { otherwise }\end{cases}
$$

After leasing all the available channels, the auctioneer earns a revenue, $\mathcal{R}$, which includes the total price paid by the winner SUs and can be given by Equation (4).

$$
\mathcal{R}=\sum_{i=1}^{N} p_{i}
$$

The proposed approach tries to enhance the spectrum utilization, $\mathcal{S}_{u}$, where $\mathcal{S}_{u}$ can be interpreted as the sum of the winning bid values from the SUs for all the assigned channels. With the channel allocation $A=\left\{a_{i j}\right\}_{N \times M}$ obtained from the bids submitted to the auctioneer, the spectrum utilization, $\mathcal{S}_{u}$, can be given by Equation (5).

$$
\mathcal{S}_{u}=\sum_{j=1}^{M} \sum_{i=1}^{N} a_{i j} b_{j i}
$$

Figure 2 shows a diagrammatic representation of the system model, where three channels, $C 1, C 2$, and $C 3$, are auctioned among eight SUs, $S 1$ to $S 8$. Following the sequentialbidding strategy, when channel $C 1$ is to be allocated, the auctioneer receives bids from $S 1, S 2, S 3$, and $S 5$ and thereby assigns the channel to $S 1$ and S3. For channel C2, even though $S 1$ finds $C 2$ to be available, it cannot bid for $C 2$ since it has already been allocated the channel C1. Similarly, S1, S2, S3, and S7 cannot bid for C3.

To provide an efficient channel allocation, the proposed approach aims at maximizing the spectrum utilization across the network. Therefore, the problem for efficient channel allocation can be stated as an optimization problem as shown in Equation (6). 


\section{Maximize}

$$
\mathcal{S}_{u}=\sum_{j=1}^{M} \sum_{i=1}^{N} a_{i j} b_{j i}
$$

subject to

$$
\begin{aligned}
& \text { (i) } a_{i j}=0 \text {, if } c_{i j}=0 \\
& \text { (ii) } a_{i j}+a_{k j} \leq 1, \text { if } x_{i k}=1 \\
& \text { (iii) } \sum_{j=1}^{M} a_{i j} \leq 1, \forall i \\
& \text { (iv) } \sum_{i=1}^{N} a_{i j}=n, 0 \leq n \leq N, \forall j \\
& a_{i j} \in\{0,1\} ; i, k \in \mathcal{N} ; j \in \mathcal{M}
\end{aligned}
$$
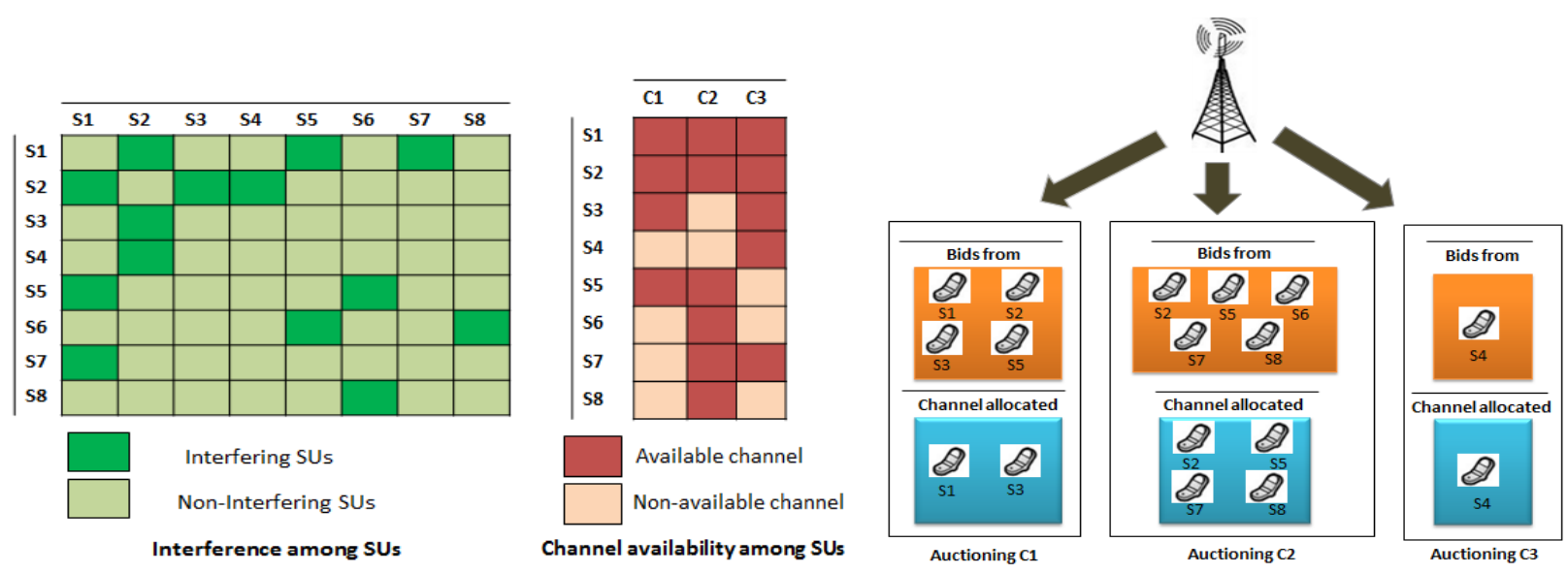

Figure 2. Diagrammatic representation of system model.

\section{Auction Properties}

The auction properties that are used for the single-sided auctions are as follows:

1. Individual rationality: An auction is individually rational if no winning bidder pays a price that is higher than the bid value submitted for the auctioned item that it won. This implies that every winner will have a non-negative utility. Thus, when $a_{i j}=1$, the winning SU $i$ pays a payment $p_{i}$ where $b_{j i} \geq p_{i}$.

2. Truthfulness: An auction is truthful if no bidder can improve its utility by submitting a bid value that is different from its true valuation, $b_{j i} \neq v_{j i}$, no matter how other bidders bid for the item. Truthfulness is important to avoid market manipulations of the bid values to ensure an efficient auction. Bidding the true private valuation maximizes the bidders' utility.

\subsection{Challenges}

This section discusses the issues that arise when CR constraints are excluded in the spectrum-auction model, and the second-price auction for multi-winner allocation. 


\subsubsection{Dynamics in SOPs}

In a CRN, every SU may not sense all the free channels. A channel available at one SU may become unavailable at another SU. This occurs due to the different hardware constraints faced by an SU during the sensing process [43]. Such a constraint also affects the channel-allocation process because if an SU bids for a channel that is not available with it and, going by the winner-determination strategy, if the SU wins the channel, then the channel will again remain unused, as the SU cannot perform any transmission. This degrades the spectrum utilization as well as the network throughput. Previously designed auction models for CRNs consider that an SU bids for all channels without having any prior knowledge on the channel availability of the SU. Additionally, some work considers that all the channels are available to each $\mathrm{SU}$, which is generally not a practical scenario in CRNs. Thus, to absorb such a CR constraint, an SU has to initially sense its available channels before starting the bidding process.

\subsubsection{Channel-Availability Time}

In a CRN, a channel allocated to an SU may become unavailable due to the return of the PU who leased the channel. Such a situation disturbs the transmission process of the SU. In an auction model, to avoid bidding for such channels, the SU needs to study the channel-availability time of every available channel. Thereafter, the SU bids for the channel only when its availability time is more than the time that the SU requires to transmit over the channel. None of the existing auction models for CRNs have incorporated this constraint while modelling the channel-allocation problem. Due to this, a winner SU may have to vacate its channel before completing its transmission, and this thereby results in a wastage of the assigned radio spectrum. Hence, excluding such a constraint can adversely affect the spectrum utilization as well as the network throughput.

\subsubsection{Second-Price Auction}

The second-price auction (or Vickrey auction) [8] provides a truthful design model for single-unit auction scenarios that allows single-winner allocation (an auctioned item given to, at most, one bidder). When the second-price auction is being deployed to lease an item, the bidder with the highest bid value wins the item and pays the highest losing bid. This guarantees an optimal and truthful allocation of the auctioned item. Now, when sequential bidding is applied for bid collection, every channel is auctioned one by one in multiple rounds, and this relates to a single-unit auction in each round, where a second-price auction can be used to obtain an optimal allocation. However, such an auction is not suitable for multi-winner allocation, where one channel can be assigned to multiple non-interfering users. Therefore, on extending the second-price auction for channel reuse, we should be able to map different non-interfering SUs to one channel. To implement a simple framework (called the general sequential auction (GSA)) with the reusability feature, we assume that for the auctioned channel, the bidder with the highest bid is declared as a winner. Then, we move to the bidder having the second-highest bid. If the bidder does not conflict with the winning SU and satisfies the allocation constraint, then they too get included in the winner list. Otherwise, the third-highest bidder is picked up. This process repeats till the last SU in the list is reached. Such a method can lead to a group of non-interfering SUs for each channel. However, here, we may not obtain the group having a high total valuation for the channel, and this can reduce the use of the spectrum bands. Thus, the challenge in our proposed model is to choose the groups of SUs that can give a high total valuation and, thereafter, improve the overall spectrum usage.

\subsection{SAMW Model}

The auction-based spectrum-allocation model SAMW tries to allocate the licensed spectrum efficiently in a CR environment. SAMW follows the procedure of the second-price auction by integrating spectrum reuse, where it maps a group of non-interfering SUs to a channel and maintains the economic properties. The PO auctions the available channels 
in decreasing order of their channel-availability times in $M$ (the total number of channels) different rounds, allowing one channel in each round. That is,

$$
T_{A(1)} \geq T_{A(2)} \geq T_{A(3)} \geq \ldots \geq T_{A(M)}
$$

An SU $i$ who cannot win the auctioned channel in the $j$ th round will decide to submit a higher bid in the $(j+1)$ th round. Bidders increase their bids so that their chances of winning the $(j+1)$ th channel increases. Moreover, such a strategy helps to improve the overall spectrum utilization along with the PO's revenue. However, in cases where $T_{A(j)}=T_{A(j+1)}$ and $b_{j i}=$ the channel capacity, the $\mathrm{SU} i$ has to submit the same bid as in the $j$ th round, i.e., $b_{(j+1) i}=$ the channel capacity. Again, when $T_{A(j)} \neq T_{A(j+1)}$ and $b_{j i}=$ the channel capacity, the $\mathrm{SU} i$ cannot bid for the $(j+1)$ th channel since $T_{A(j+1)}$ will be less that $T_{R(i(j+1))}$. For instance, let SUs, $S 1, S 2$, and $S 3$, be the three bidders in the game where interference exists between $S 1$ and $S 3$, and $S 2$ and $S 3$. Consider two channels $C 1$ and $C 2$ with channel capacities of $42 \mathrm{bps}$ and availability times $T_{A(1)}=2.60 \mathrm{~s}$ and $T_{A(2)}=2.80$ $\mathrm{s}$, respectively. Both channels are available to all three SUs. Figures 3 and 4 provide a diagrammatic view of this example. With sequential bidding, $C 2$ is auctioned in the first round, and the bids are $b_{21}=3.32, b_{22}=3.71$, and $b_{23}=4.11$. Consider that $S 3$ wins the channel $C 2$. Now, in second round, remaining SUs will increase their bid values so that they can get the channel. $b_{11}=3.69$ and $b_{12}=3.85$ are the bids for $C 1$. Thus, with multi-winner allocation, both $S 1$ and $S 2$ win $C 1$, and the spectrum utilization obtained is 11.65. Now, let us consider concurrent bidding where all the channels are auctioned simultaneously. As such, every SU bids for all its available channels, giving $b_{11}=3.16$, $b_{12}=3.87$, and $b_{13}=3.56$ for channel $C 1$ and $b_{21}=3.32, b_{22}=3.71$, and $b_{23}=4.11$ for channel C2. The SUs S1 and S2 win channel $C 1$ and the SU S3 wins channel C2, giving an overall spectrum utilization of 11.14. Therefore, with homogeneous channels, sequential bidding is preferable since the bid values increase in each round, which always increases the spectrum utilization. However, no such strategy for bid submission can be applied in concurrent bidding. In the proposed model, on auctioning a channel $j$ in the $j$ th round, a bid vector $B_{j}$ gets formed and the PO sets a reserve price $r_{j}$ based on the bids in $B_{j}$. Finally, when a channel is allocated to the winner SUs, the SUs and the channel can no longer take part in the further allocation process.

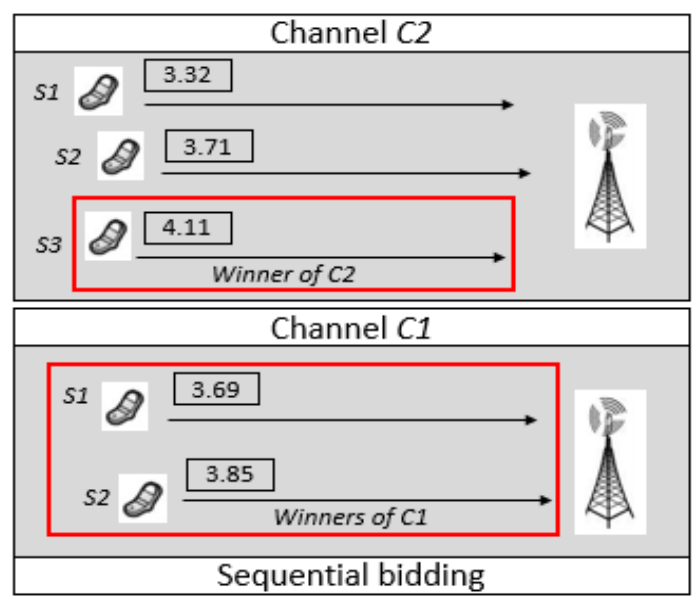

Figure 3. Sequential bidding for homogeneous channels. 


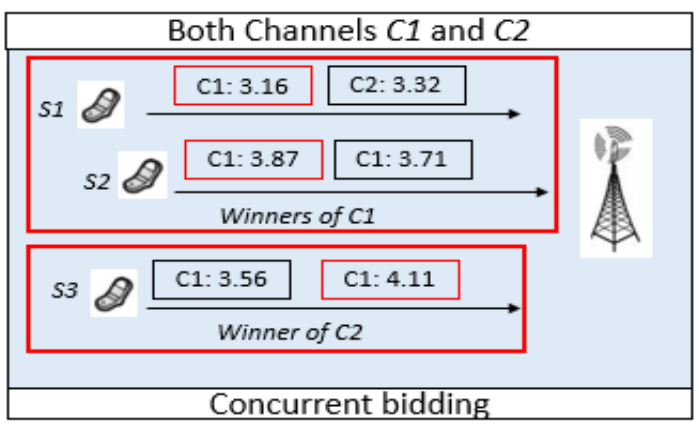

Figure 4. Concurrent bidding for heterogeneous channels.

Three different algorithms are carried out to solve the channel-allocation problem in the SAMW model. They are the group-formation algorithm, spectrum-allocation algorithm, and pricing algorithm. Figure 5 shows a flowchart representation of the proposed auction process, which implements the three algorithms on auctioning each channel. Initially, on starting the auction for a channel $j$, SUs decide their respective valuations for the channel and thereafter submit their bid values according to Equation (1). Furthermore, the three algorithms are carried out to decide the winners and the payment for the channel.

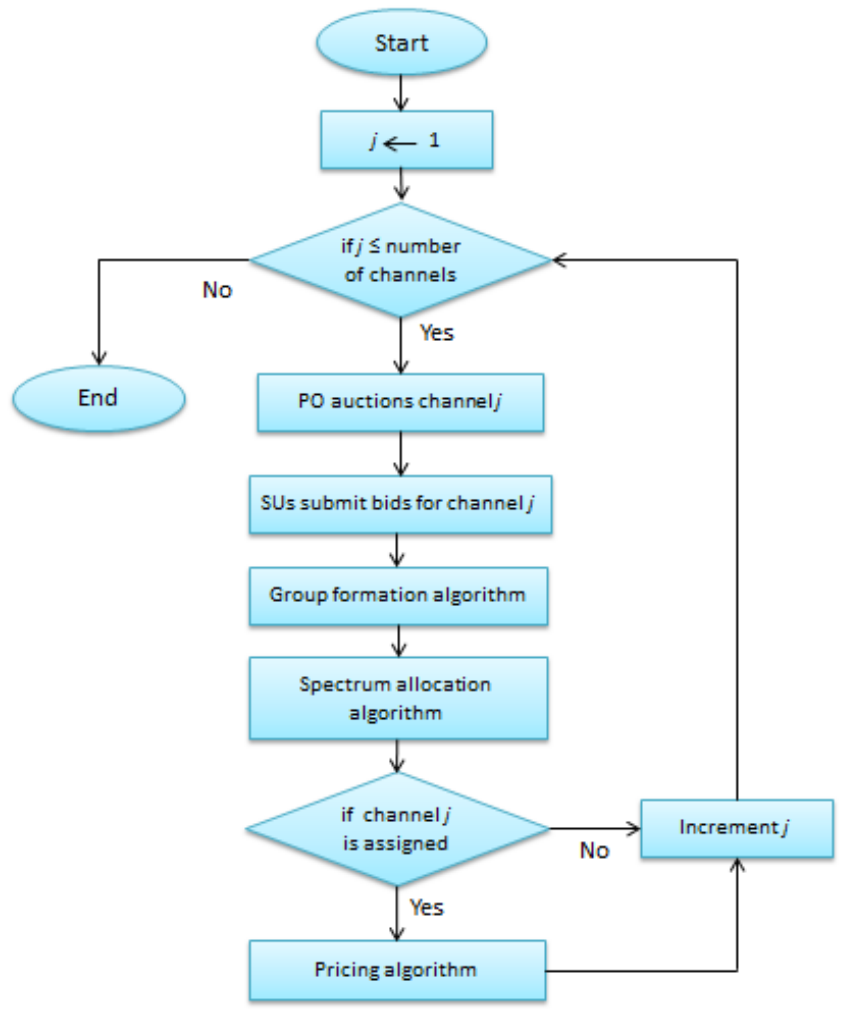

Figure 5. Flowchart representation of the proposed auction process.

\subsubsection{Group-Formation Algorithm}

This algorithm (Algorithm 1) forms groups of non-interfering SUs such that a common channel can be assigned to a group. It is executed for every auctioned channel one by one, where a winning bid value and a winner set of SUs are computed for every SU who bids for the channel. This constructs a winning-value vector, $\mathcal{W}$, and a winner-group vector, $\mathcal{S}$, which holds the winning bid value and winner set of SUs, respectively, for every SU bidding for the channel. Now, on auctioning a channel $j$, every SU computes a winnerdeciding matrix, $Z$, one by one, given as $Z=\left\{z_{i k}\right\}_{|\mathcal{N}| \times|\mathcal{N}|}$, where for the $i$ th row, $z_{i k}=z_{i l}=1$ 
implies that SUs $k$ and $l$ can be taken together for assigning the auctioned channel. For each SU $i \in \mathcal{N}$, matrix $Z$ is initialized to 0 , after which different $S U$ combinations that include $\mathrm{SU} i$ are computed for the auctioned channel $j$ while taking into account the interference and channel-availability constraints. Then, from $Z$, the SU combination that gathers the highest total valuation for the given channel is considered for further action. However, if ties occur for the total valuation, any one of the SU combinations is randomly picked. A winning-value vector $\mathcal{W}=\left\{w_{i}\right\}_{1 \times|\mathcal{N}|}$ is built up, where $w_{i}$ is the highest total valuation obtained from $Z$ for $\mathrm{SU} i$, and $w_{i}=0$ when $b_{j i}=0$, which implies that $\mathrm{SU} i$ has no bid for channel $j$. To begin with, $w_{i}$ is initialized to $0, \forall i \in \mathcal{N}$. Additionally, a winner-group vector $\mathcal{S}=\left\{s_{i}\right\}_{1 \times|\mathcal{N}|}$, where $s_{i} \subseteq\{1,2,3, \ldots, N\}$, is constructed that holds the SU combination corresponding to the valuation entered in $w_{i}$. This implies that every element of $\mathcal{S}$ is itself a vector of SUs, where initially, $\forall i \in \mathcal{N}, s_{i}$ is taken to be empty. Once the algorithm completes, we get the groups that can participate in the auction process for channel $j$. For the computational complexity of Algorithm 1, we find that for every ith iteration of the outermost loop, the two inner loops run $N$ times each. For every $q$ th iteration of the first inner loop, there appears another inner loop that runs $N$ times. Then, for every $k$ th iteration, there can be $(N-1)$ runs to check for interference conditions in $l \in S U L i s t$ in the algorithm. Similarly, for every gth iteration of the second inner loop, another innermost loop runs $N$ times. Hence, the overall time complexity for Algorithm 1 is computed to be $O\left(N^{4}\right)$.

\subsubsection{Spectrum-Allocation Algorithm}

With Algorithm 2, spectrum allocation is carried out for the auctioned channel. After $w_{i}$ values are determined for all the SUs who bid for the channel $j$, we implement Algorithm 2, which picks the SU combination corresponding to the maximum total valuation in $\mathcal{W}$, and this accordingly updates the allocation matrix for channel $j$. For the computational complexity, there can be $N$ runs for updating the allocation matrix, giving the time complexity as $O(N)$ for Algorithm 2.

\subsubsection{Pricing Algorithm}

On acquiring the channel $j$, every winning $\mathrm{SU} i$ needs to pay a price $p_{i}$ to the auctioneer. If $\mathrm{SU} i$ remains unassigned, then $p_{i}=0$. Otherwise, to compute $p_{i}$, Algorithm 3 is executed. A group of SUs who are interfering with SU $i$ but not with other winners of channel $j$ are gathered in $\mathcal{D}$. $\widetilde{B_{j}}$ holds the respective bids $b_{j k}, \forall k \in \mathcal{D}$. Thereafter, Algorithm 1 takes $\widetilde{B}_{j}$ and $\mathcal{D}$ as input to compute the highest total valuation from $\widetilde{W}=\left\{\widetilde{w}_{i}\right\}_{1 \times|\mathcal{D}|}$. If $\widetilde{w}_{i}=\max (\widetilde{W})$, then the payment $p_{i}=\widetilde{w}_{i}$. Otherwise, if $\nexists \mathcal{D}$ satisfies the conditions for payment, then $p_{i}$ is the reserve price, $r_{j}$, set for the channel. $O\left(N^{2}\right)$ gives the computational complexity for Algorithm 3.

In brief, the proposed auction-based approach for spectrum allocation deploys a sequential-bidding policy that tries to proffer channel reuse while restricting to channelavailability and interference and allocation constraints, and eventually trying to enhance the overall spectrum utilization across the network.

\subsection{Proof of Auction Properties}

Theorem 1. SAMW is individually rational.

Proof of Theorem 1. For the proposed mechanism to be individually rational, every winner SU $i$ has to pay a price $p_{i}$ on wining a channel $j$ such that $p_{i} \leq b_{j i}$ when $a_{i j}=1$. According to the payment strategy in Algorithm 3, SU $i$, on wining channel $j$, pays a price $p_{i}$ from $\mathcal{D}$. There appears a group of SUs, $\mathcal{G}$, in $\mathcal{D}$, such that $p_{i}=\sum_{\forall h \in \mathcal{G}} b_{j h}$. Here, $\forall h \in \mathcal{G}, x_{i h}=1$ and $x_{f h}=0 \forall f \in \mathcal{N}$, where $f$ is a winner SU of the channel $j$ other than SU $i$. Moreover, $\sum_{\forall h \in \mathcal{G}} b_{j h}$ gives the highest total valuation in $\mathcal{D}$ for channel $j$, where $\sum_{\forall h \in \mathcal{G}} b_{j h} \leq b_{j i}$. Otherwise, if there is no such $\mathcal{D}$ to get the payment, then $p_{i}$ is the reserve price and $b_{j i} \geq r_{j}$ according to Equation (2). As such, the $p_{i}$ from SU $i$ for channel $j$ cannot be greater than bid $b_{j i}$, which in turn provides non-negative utility to $\mathrm{SU} i$, i.e., $u_{i} \geq 0$. 
Lemma 1. Given $B_{j}=\left(b_{j 1}, b_{j 2}, \ldots, b_{j i}, \ldots, b_{j N}\right)$, bids submitted by SUs on auctioning channel $j$. If SU $i$ wins the auction for channel $j$ by bidding $b_{j i}$, then it also wins the channel by bidding $b_{j i}^{\prime}>b_{j i}$ (provided that all other conditions remain same).

Proof of Lemma 1. On auctioning channel $j$, an SU $i$ wins the channel by bidding $b_{j i}$, which incurs the highest total valuation along with the other non-interfering winners for the channel. If $\exists b_{j k}=b_{j i}$ such that $a_{k j}=0$, this implies that $x_{i k}=1$ (other winners are non-conflicting with SU $k$ ). Otherwise, if $b_{j k}>b_{j i}$, then SU $i$ cannot win with $b_{j i}$. Thus, submitting $b_{j i}^{\prime}>b_{j i}$ will again allow SU $i$ to win the channel. Additionally, if $\exists b_{j k} \geq b_{j i}$ such that $a_{k j}=0$, this implies that SU $k$ interferes with some winner SU of channel $j$ (other than $\mathrm{SU} i$ ), which would a provide lesser or equal valuation for the channel. On bidding $b_{j i}^{\prime}>$ $b_{j i}$, SU $i$ still wins the channel $j$ irrespective of $b_{j i}^{\prime} \geq b_{j k}$ or $b_{j i}^{\prime}<b_{j k}$ since the inclusion of $b_{j i}^{\prime}$ among the winning bid values increases the highest total valuation for the channel.

Theorem 2. SAMW is truthful.

Proof of Theorem 2. Let $u_{i}$ and $u_{i}^{\prime}$ represent the utility when SU $i$ bids its true value $v_{j i}$ and an untruthful bid $b_{j i} \neq v_{j i}$ for a channel $j$, respectively. The proposed mechanism is truthful when no SU $i$ can improve its utility $u_{i}$ by submitting a bid value $b_{j i} \neq v_{j i}$ for channel $j$, i.e., $u_{i} \geq u_{i}^{\prime}$ (provided that all other conditions remain same).

Case 1: $b_{j i}>v_{j i}$

By Lemma 1 , it cannot be true that bidder $i$ wins the auction by bidding $v_{j i}$ but loses when it bids $b_{j i}$ since $b_{j i}>v_{j i}$. Thus, the three subcases that can arise are:

(1) When SU $i$ loses the game by bidding both $b_{j i}$ and $v_{j i}$; the utility obtained is $u_{i}=u_{i}^{\prime}=0$ as per Equation (3).

(2) SU $i$ wins on bidding $b_{j i}$ but loses for $v_{j i}$, which gives a utility $u_{i} \geq u_{i}^{\prime}$. With $p_{i}$ and $p_{i}^{\prime}$ being the payment for $v_{j i}$ and $b_{j i}$ respectively, $p_{i}=u_{i}=0$. For $v_{j i}$ to lose, there can be a situation where $\exists b_{j k} \geq v_{j i}$ and $x_{i k}=1$, such that the total valuation including $b_{j k}$ is higher than or equal to that including $v_{j i}$. Additionally, there can be another situation where there appears a group of SUs, $\mathcal{G}$, such that $\forall h \in \mathcal{G}, x_{i h}=1$, and $b_{j h}<$ $v_{j i}$, but $\sum_{\forall h \in \mathcal{G}} b_{j h} \geq v_{j i}$. Since $b_{j i}>v_{j i}$ wins the game, this implies that $p_{i}^{\prime}=b_{j k}$ or $p_{i}^{\prime}=$ $\sum_{\forall h \in \mathcal{G}} b_{j h}$, following Algorithm 3 . Hence, this gives $u_{i} \geq u_{i}^{\prime}$.

(3) When SU $i$ wins by bidding both $b_{j i}$ and $v_{j i}$, this provides $u_{i}=u_{i}^{\prime}$. With $v_{j i}$ winning the game, there may appear a bid $b_{j k} \geq v_{j i}$ and $x_{i k}=0$ such that in one situation, SU $k$ wins the channel $j$ along with SU $i$, whereas in another situation, SU $k$ cannot win the channel $j$ even when $x_{i k}=0$ because SU $k$ interferes with a winner SU of channel $j$ other than $\mathrm{SU} i$, which yields a higher total valuation for the channel. On bidding $b_{j i}$, SU $i$ still wins, giving a payment $p_{i}^{\prime}=p_{i}$, where $p_{i}$ is the payment for $v_{j i}$, since the highest valued group in $\mathcal{D}$ is less than or equal to $v_{j i}$. Additionally, with $b_{j k}=v_{j i}$ and $x_{i k}=1$, when SU $i$ wins the channel and SU $k$ loses, $p_{i}=b_{j k}$ if SU $k$ is not interfering with other winners of channel $j$. Otherwise, $p_{i} \leq b_{j k}$. With $b_{j i}$, SU $i$ wins by paying $p_{i}^{\prime}=p_{i}$, giving $u_{i}=u_{i}^{\prime}$.

Case 2: $b_{j i}<v_{j i}$

By Lemma 1, it cannot be true that bidder $i$ wins the auction by bidding $b_{j i}$ but loses when it bids $v_{j i}$ since $v_{j i}>b_{j i}$. Thus, the three subcases that can arise are:

(1) For proving that $\mathrm{SU} i$ loses the game by bidding both $b_{j i}$ and $v_{j i}$, the same analysis as provided in Case 1 is used, which gives $u_{i}=u_{i}^{\prime}=0$.

(2) When SU $i$ wins with $v_{j i}$ and loses with $b_{j i}, p_{i}^{\prime}=u_{i}^{\prime}=0$. However, according to Theorem $1, u_{i} \geq 0$, which states that $u_{i} \geq u_{i}^{\prime}$.

(3) When SU $i$ wins by bidding both $b_{j i}$ and $v_{j i}$, a similar situation arises as discussed in Case 1 . However, for $b_{j i}$ to win, $b_{j i} \geq p_{i}$, where $p_{i}$ is the payment for $v_{j i}$. This gives $p_{i}^{\prime}=p_{i}$, and accordingly, $u_{i}=u_{i}^{\prime}$. 


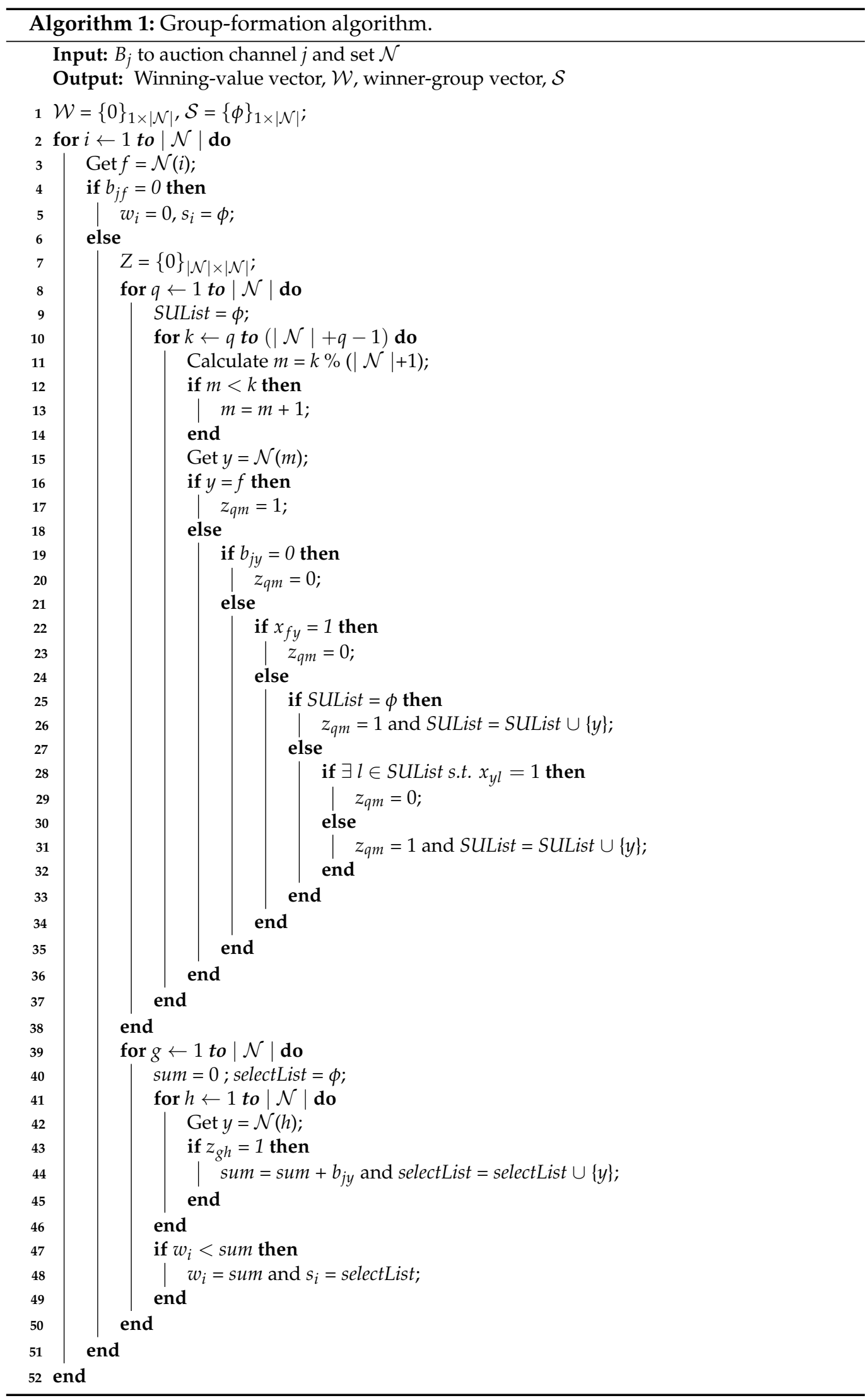



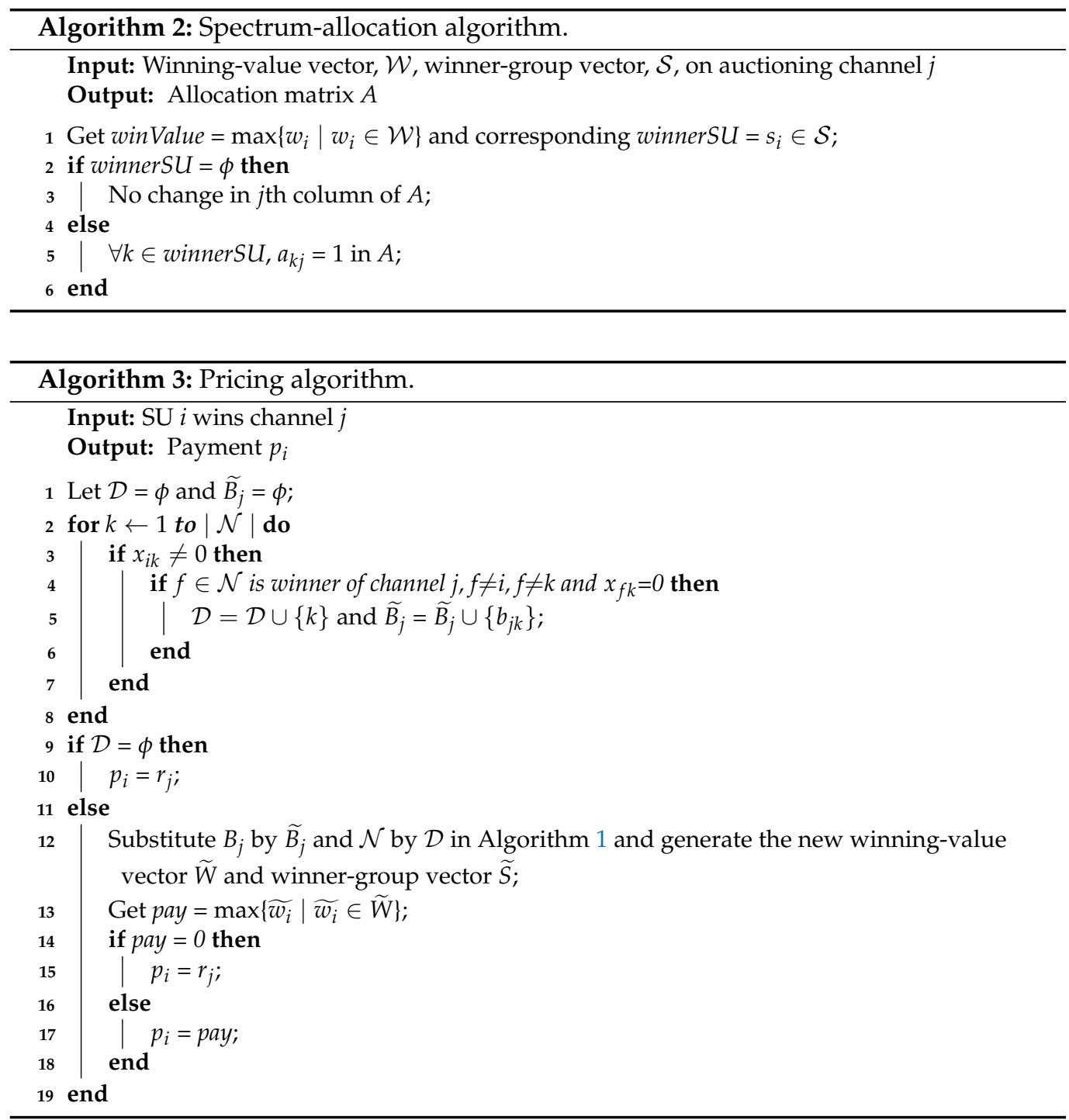

This completes the proof that a bidder cannot improve its utility by submitting a bid value that is different from the true valuation.

\section{Performance Evaluation}

To evaluate the performance of SAMW, a MATLAB-based simulation was carried out as described in this section. We consider a CR environment with a PO acting as the auctioneer, who handles multiple bidders (SUs) that are randomly distributed in an area of size $800 \times 800 \mathrm{~m}$. We assume that the sensing data are obtained through an available robust sensing process, and this provides the set of free channels to each SU. Interference among the SUs was modelled by applying the distance-based criterion [44]. The channels available were taken to be GSM channels with a bandwidth of $200 \mathrm{KHz}$ and channel capacity of $270 \mathrm{Kbps}$. First, we examined the proposed approach, SAMW, by comparing it with a general sequential auction (GSA), which applies a second-price auction with channel reuse as described in Section 3.2.3. Additionally, we compared SAMW with VERITUS [31]. VERITUS was chosen among the existing approaches since it is a singlesided model that also aims at improving the spectrum utilization, identically to SAMW. We considered that VERITUS abides by the network constraints and accordingly gathers the bids. However, in VERITUS, bids are submitted at once for all the channels since the channels are auctioned concurrently. Additionally, each SU will request only one channel according to the allocation constraint. Two different network scenarios were deployed, 
where in one scenario, the number of channels was varied as $\{4,6,8,10,12,14\}$, keeping the number of SUs unchanged at 40, and in the other scenario, the number of SUs was varied as $\{20,30,40,50,60\}$, keeping the number of channels unchanged at 6 . Then, we also explored the confidence intervals at the 95\% confidence level for all the scenarios. Second, we compared SAMW (which allows spectrum reuse) with a simple second-price auction, which follows single-winner allocation. This explored the change in spectrum utilization with and without spectrum reuse. Third, to show the influence of the dynamics in SOPs and differences in channel-availability time in CRNs, we compared our model with VERITUS, where in VERITUS, none of the network constraints were incorporated (as in the actual model) to frame the model. For each type of scenario, we ran the simulation for 500 rounds to take the average values for each performance metric.

The performance metrics used for evaluating SAMW are as follows:

- Average utility, $\left(A v g_{u}\right)$ : This is the ratio of utility obtained by all the winning SUs to the total number of participating SUs, as expressed in Equation (7).

$$
A v g_{u}=\frac{\sum_{i=1}^{N} u_{i}}{N}
$$

- User satisfaction $\left(U_{s}\right)$ : This is the ratio of the winner SU count for all the available channels to the total number of participating SUs, as expressed in Equation (8).

$$
U_{s}=\frac{\sum_{j=1}^{M} \sum_{i=1}^{N} a_{i j}}{N}
$$

- $\quad$ Spectrum utilization $\left(\mathcal{S}_{u}\right)$ : This computes the total winning bids from the winners as expressed in Equation (5).

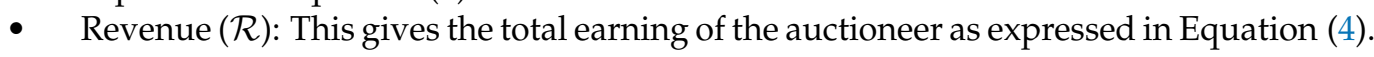

- Successful user ratio $\left(\mathcal{S}_{r}\right)$ : This metric is used to show how the two network constraints can have an affect on the performance of the designed model. $\mathcal{S}_{r}$ is the ratio of the number of winning SUs who can complete their transmission to the total number of winning SUs in the model.

$$
\mathcal{S}_{r}=\frac{\sum_{\substack{i=1 \\\left(T_{A(j)}>T_{R(i j))} \wedge\left(c_{i j}=1\right)\right.}}^{M} a_{i j}}{\sum_{j=1}^{M} \sum_{i=1}^{N} a_{i j}}
$$

\section{Discussion}

In Figure 6a-d, we show the plots for the performance metrics obtained by varying the number of channels. In Figure $7 \mathrm{a}-\mathrm{h}$, the confidence intervals for the performance metrics shown were obtained at the $95 \%$ confidence level by considering two different cases, four channels with 40 SUs and 12 channels with 40 SUs. These two cases represent the upper limit and the lower limit, respectively, for the graphs Figure 6a-d along the $x$-axis. From Figures $6 a$ and $7 a, b$, we can observe that with an increase in the number of channels, the spectrum utilization increases for all the approaches. This is due to the greater availability of spectrum resources, which allows a higher number of users to access the spectrum. The performance of the GSA is degraded as compared to that of SAMW because in the GSA, when a channel is auctioned, it starts by picking the highest-bidding SUs sequentially while restricting to network constraints. However, such an approach may not provide the highest-valued group for each channel, and this thereby decreases the use of the spectrum in the GSA. However, when the number of channels increases and gets closer to the number of SUs, then the GSA can outperform SAMW in certain cases. This occurs because a bidder who is chosen as a winner for a channel during SAMW may not be considered as a winner on auctioning the same channel with the GSA. This bidder then submits a higher valuation 
for another channel in the GSA, which when acquired by the bidder, improves the spectrum utilization. Additionally, VERITUS shows a decrease in its performance as compared to SAMW because in VERITUS, bids for all the available channels are collected concurrently, and the allocation process proceeds without considering the highest-valued group for every channel. Similarly, the revenue earned by the auctioneer increases with an increase in the number of channels as shown in Figures $6 \mathrm{~b}$ and $7 \mathrm{c}, \mathrm{d}$ since a higher number of items are now available for auction. The payment algorithms are different in all three approaches. In the GSA, we pick the next-highest bidder for payment. In VERITUS, the next-highest bidder is picked while taking into account certain constraints in the model. In SAMW, we follow Algorithm 3 for payment, where SUs interfering with the winner are taken along with other different considerations. Thus, the revenue earnings with SAMW greatly depend on the interference status of the users in the network. Now, going by the payment algorithm in SAMW, we can find several winning bidders paying the reserve price, which thereby deteriorates the revenue in SAMW as compared to in the GSA and VERITUS. However, the use of the reserve price in SAMW helps the auctioneer to earn at least a minimal payment (always greater than zero) for each leased channel, which further improves the overall revenue. From Figures $6 \mathrm{c}$ and $7 \mathrm{e}, \mathrm{f}$, we find that SAMW shows a greater improvement in average utility as compared to the other two approaches. This is accounted for by the payment algorithm taken up in SAMW, which creates high utility values (Equation (3)) amongst the winners. Similarly, user satisfaction shows a rise in performance as observed from Figures $6 \mathrm{~d}$ and $7 \mathrm{~g}$, h due to the greater availability of channels. When the number of channels is greater, a higher number of SUs can use the spectrum for their transmission. SAMW shows good results due to the allocation method applied in SAMW as compared to GSA and VERITUS.

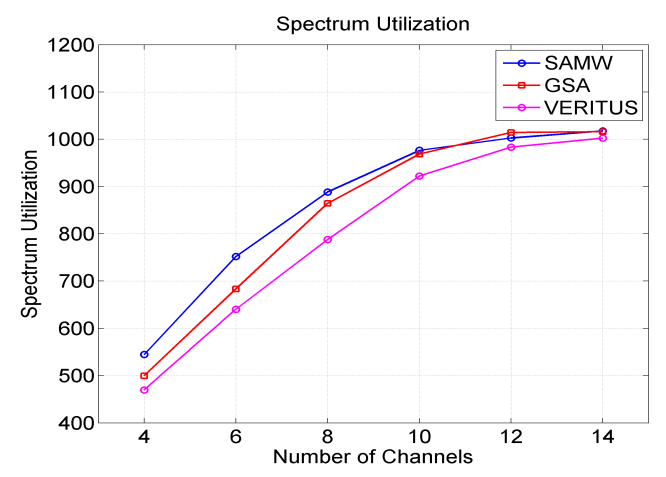

(a) Spectrum utilization with respect to number of channels

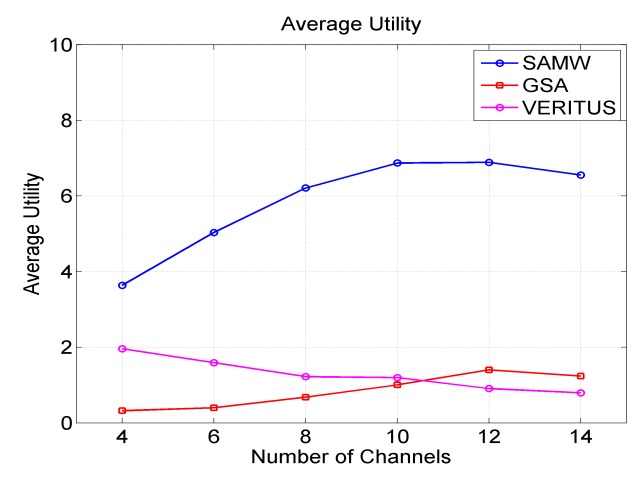

(c) Average utility with respect to number of channels

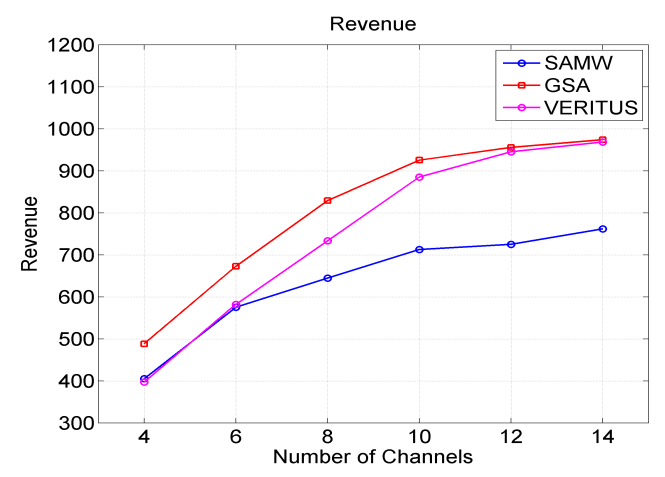

(b) Revenue with respect to number of channels

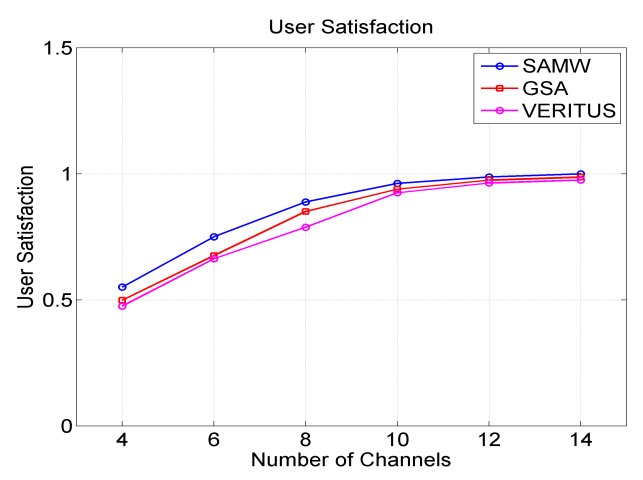

(d) User satisfaction with respect to number of channels

Figure 6. Spectrum utilization, revenue, average utility, and user satisfaction for sequential auction-based multi-winner (SAMW), general sequential auction (GSA), and VERITUS with respect to number of channels. 


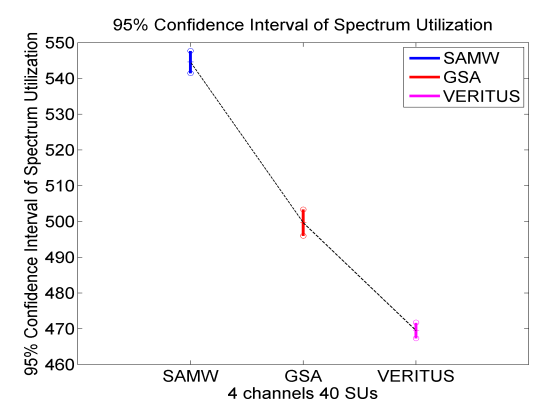

(a) Spectrum utilization for 4

channels with 40 secondary user

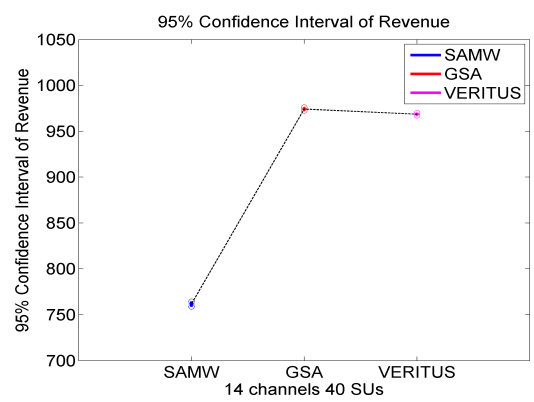

(d) Revenue for 14 channels with 40 secondary user

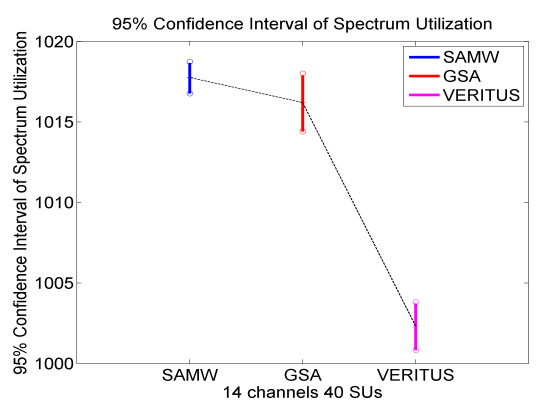

(b) Spectrum utilization for 14

channels with 40 secondary user

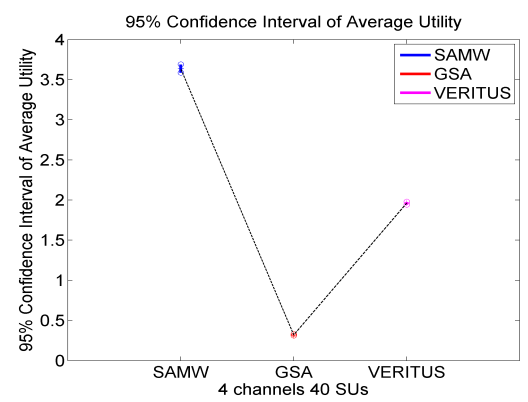

(e) Average utility for 4

channels with 40 secondary user

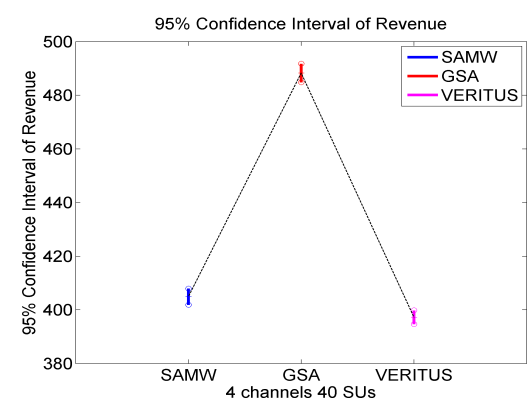

(c) Revenue for 4 channels with 40 secondary user

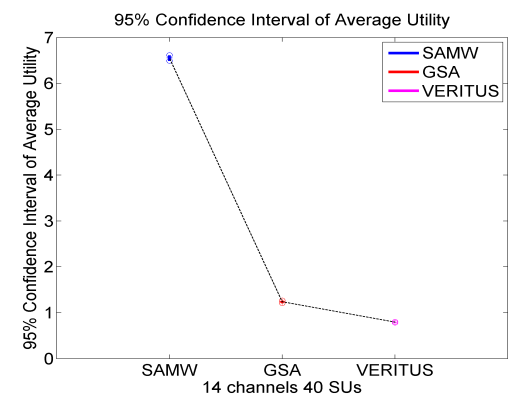

(f) Average utility for 14 channels with 40 secondary user

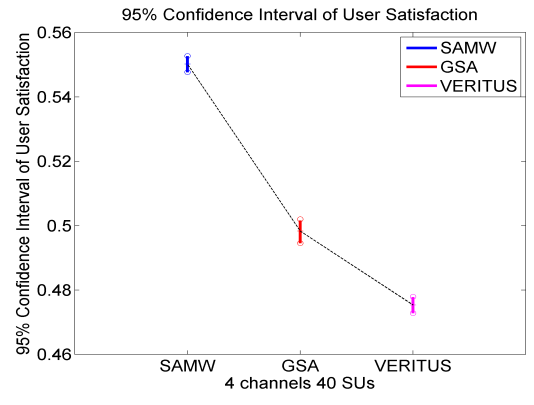

(g) User satisfaction for 4 channels with 40 secondary user

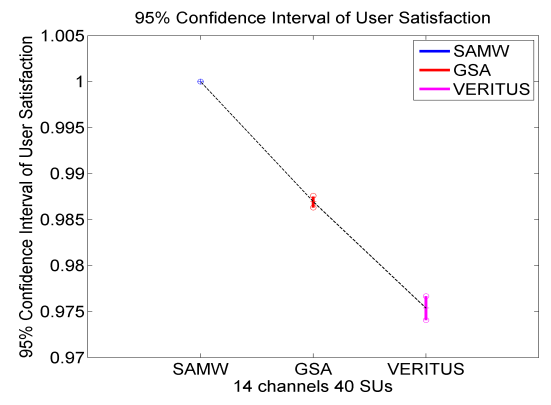

(h) User satisfaction for 14 channels with 40 secondary user

Figure 7. Confidence intervals at $95 \%$ confidence level for spectrum utilization, revenue, average utility and user satisfaction for 4 channels with 40 secondary users (SUs) and 14 channels with 40 SUs

Figure $8 \mathrm{a}-\mathrm{d}$ display the plots for the performance metrics when we vary the number of SUs. Corresponding to these plots, we obtained the confidence intervals for the performance metrics at the 95\% confidence level as shown in Figure 9a-h. Two different cases were considered while computing the confidence interval, 20 SUs with six channels and 60 SUs with six channels. These two cases represent the upper limit and the lower limit, respectively, for the graphs Figure 8a-d along the $x$-axis. In Figures $8 \mathrm{a}$ and $9 \mathrm{a}, \mathrm{b}$, the spectrum utilization shows a rise in its performance upon increasing the count of SUs. This is because, with an increase in the number of SUs, there appears a higher number of bidders who can acquire the auctioned channel, and along with that, the range of bids gathered for the auctioned channel gets wider. However, the use of spectrum is comparatively less in the GSA and VERITUS. This is accounted for by the allocation methods deployed for both the approaches, where they do not look for the highest-valued group of bidders for each channel. Similarly, the revenue improves in all the three approaches since the 
number of buyers gets increased for an auctioned item. However, due to the payment algorithm designed in SAMW, the revenue gathered in SAMW is computed to be less compared to that for the GSA and VERITUS, which can be seen from Figures $8 b$ and $9 c, d$. Then, from Figures $8 c, d$ and $9 \mathrm{e}-\mathrm{h}$, we can observe that the average utility and user satisfaction curves tend to decline with an increasing number of SUs. This is because, for same number of channels, more SUs claim to get access to the channel, incurring an increase in competition amongst the users. However, this decline appears to be moderate due to the implementation of channel reuse in the model. A performance comparison shows that SAMW outperforms the other two approaches to achieve good results in both the metrics.

In Figures 10a,b and 11a,b, SAMW is compared with the second-price auction, which allows single-winner allocation for every auctioned channel. SAMW performs channel reuse where multiple non-interfering SUs can be simultaneously given a common channel. However, in the other approach, one channel can be assigned to only one bidder, whoever bids the highest. Under the two network scenarios deployed for simulation, we can infer that both the spectrum utilization and revenue show much improved performance for SAMW due to its reusability feature.

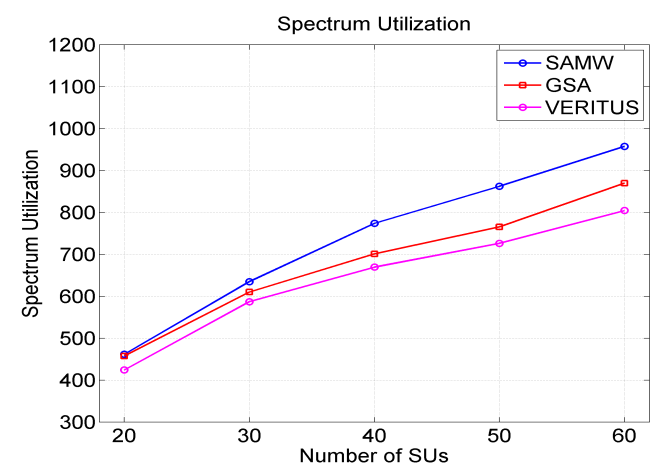

(a) Spectrum utilization with respect to number of secondary users

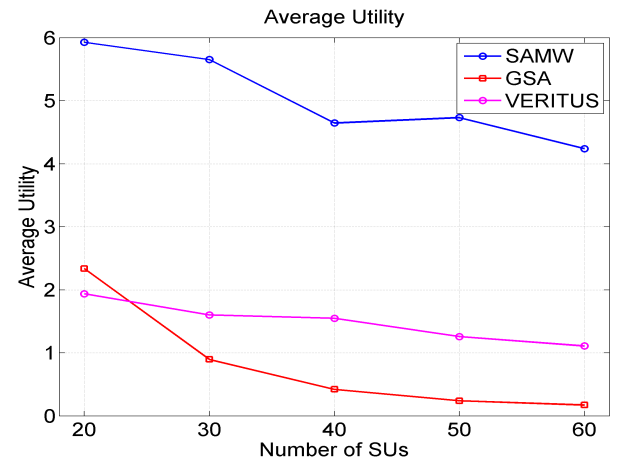

(c) Average utility with respect to number of secondary users

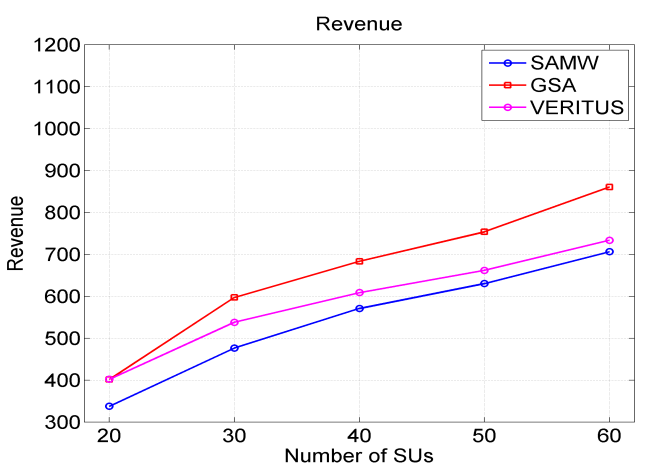

(b) Revenue with respect to number of secondary users

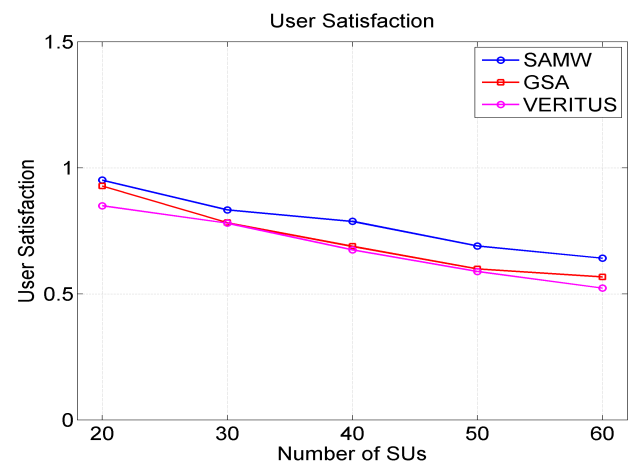

(d) User satisfaction with respect to number of secondary users

Figure 8. Spectrum utilization, revenue, average utility, and user satisfaction for SAMW, GSA, and VERITUS with respect to number of secondary users.

Then, to show the impact of the two network constraints, i.e., the dynamics in the SOPs of SUs and varying availability time of channels, we obtained the curves shown in Figure 12a,b. Here, we simulated VERITUS such that it did not include any of the network constraints, according to [31]. Then, when we performed channel allocation in VERITUS, there could be some winning bidders who could not complete their transmission due to the constraints arising in the CRN. However, since SAMW makes up the model by incorporating such constraints, in SAMW, every winner SU can complete its transmission 
over the assigned channel, which can be viewed in both the figures. Accordingly, the spectrum utilization improves in SAMW because there is no wastage of the spectrum.

Hence, the results obtained through the network simulations state that the proposed auction model SAMW proffers an improved utilization of the unused radio spectrum, which in turn helps to settle the spectrum-scarcity problem. For the practical implementation of the auction model, we can use CR-related standards such as IEEE 802.22 (Wireless Regional Area Network) [45], IEEE 802.11af (Wi-Fi over TV whitespace) [46], etc. There should be practical infrastructural support and spectrum usage policy for running auctioneers, bidders, and auction centre functionality in the deployed network environment.

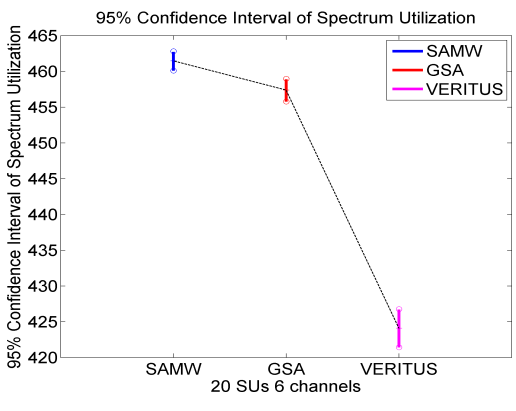

(a) Spectrum utilization for 20 secondary users with 6 channels

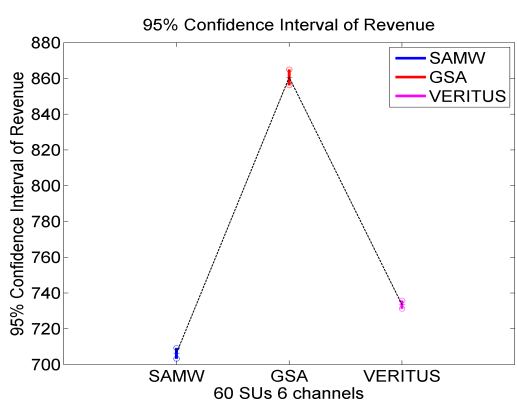

(d) Revenue for 60 secondary users with 6 channels

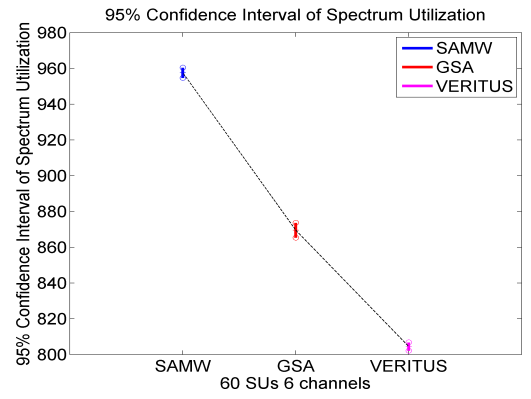

(b) Spectrum utilization for 60 secondary users with 6 channels

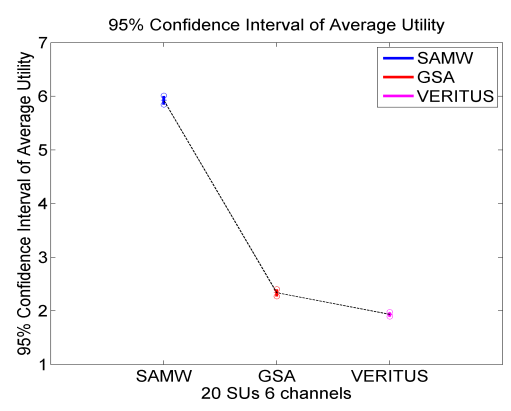

(e) Average utility for 20 secondary users with 6 channels

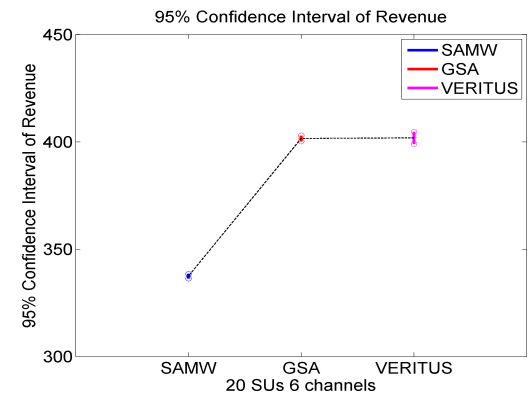

(c) Revenue for 20 secondary users with 6 channels

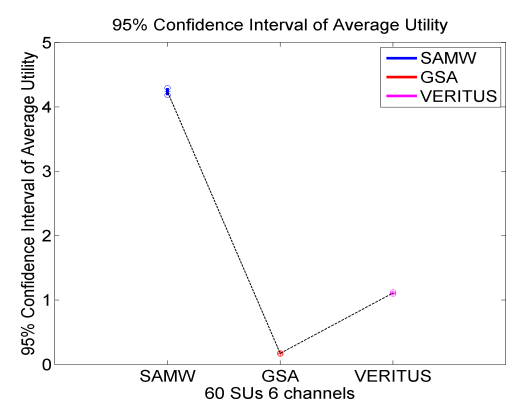

(f) Average utility for 60 secondary users with 6 channels

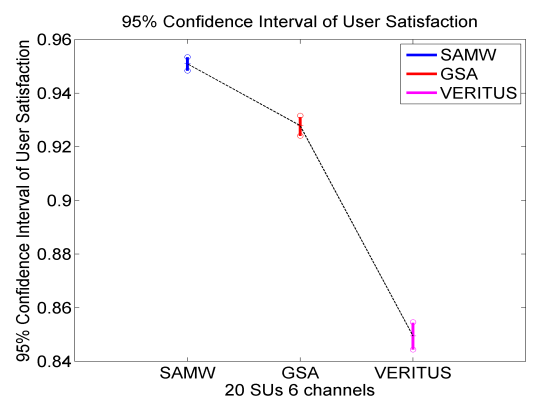

(g) User satisfaction for 20 secondary users with 6 channels

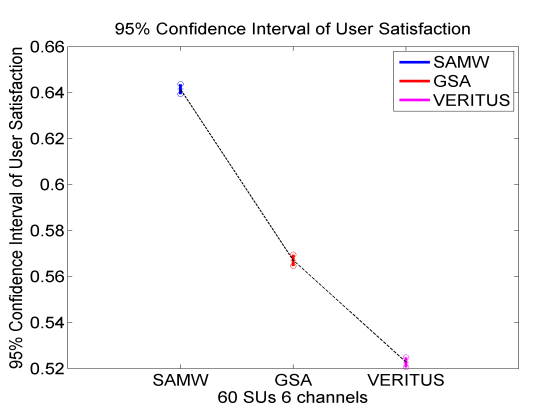

(h) User satisfaction for 60 secondary users with 6 channels

Figure 9. Confidence intervals at $95 \%$ confidence level for spectrum utilization, revenue, average utility and user satisfaction for 20 SUs with 6 channels and 60 SUs with 6 channels. 


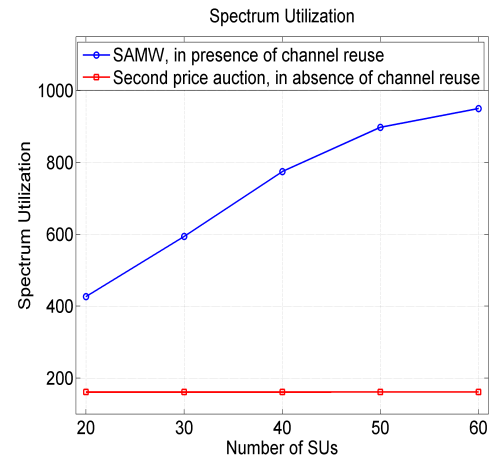

(a) Spectrum utilization with respect to number of secondary users

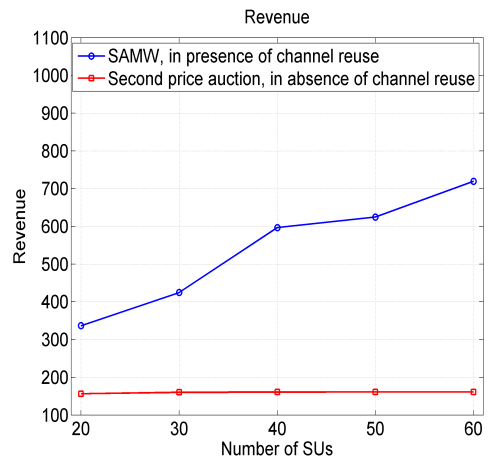

(b) Revenue with respect to number of secondary users

Figure 10. Spectrum utilization and revenue for SAMW and second-price auction with respect to number of secondary users.

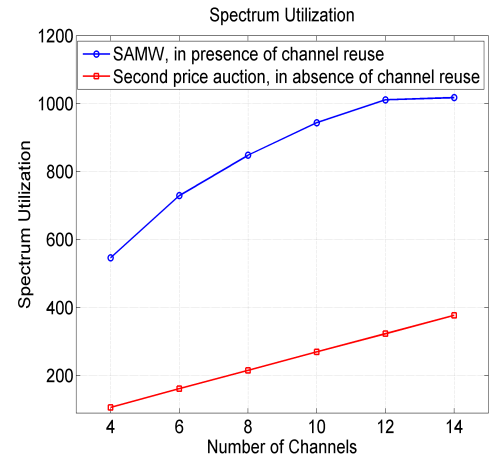

(a) Spectrum utilization with respect to number of channels

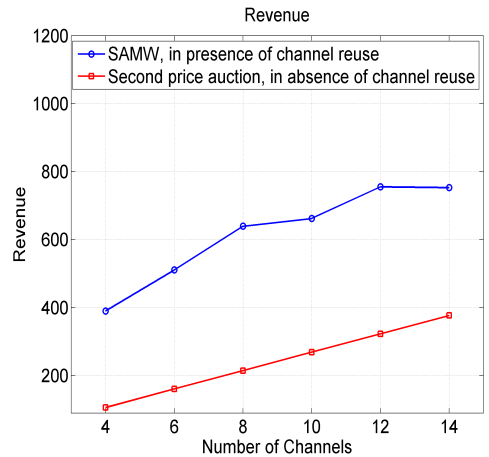

(b) Revenue with respect to number of channels

Figure 11. Spectrum utilization and revenue for SAMW and second-price auction with respect to number of channels.

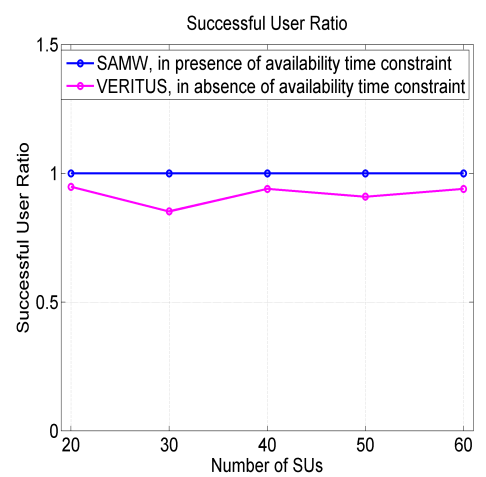

(a) Successful user ratio with respect to number of secondary users

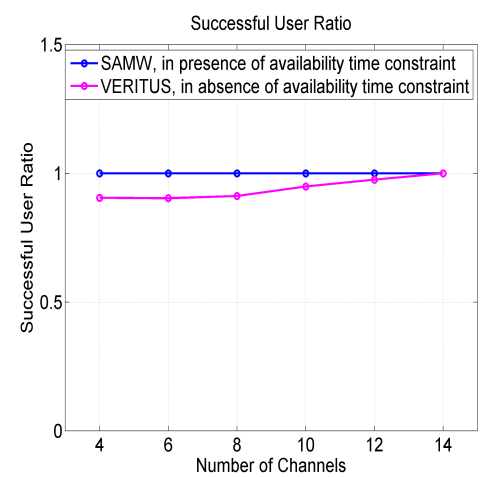

(b) Successful user ratio with respect to number of channels

Figure 12. Successful user ratio for SAMW and VERITUS.

\section{Conclusions}

In this paper, we propose SAMW, a single-sided sequential-bidding-based auction mechanism that enables spectrum reuse. On auctioning homogeneous channels, SUs offer different bid values according to their spectrum availability. Compared with existing 
research on CRNS, this model addresses two concerning issues surrounding CRNs, viz. the dynamics in the spectrum opportunities among SUs and varying availability time of channels, which further influence the bid-submission process. Winner determination and pricing strategies are carried out by the auctioneer to determine the winners and their respective payments. Truthful bidding strategies are used so that any kind of manipulation with the bid values can be avoided. Finally, the simulation results demonstrate that SAMW outperforms the GSA model as well as the VERITUS model in terms of spectrum utilization, revenue, average utility, and user satisfaction, which altogether enhance the network performance in a CRN. For future work, we would like to design a multi-channelmulti-winner-allocation scheme for CRNs by incorporating the CR constraints. Another future initiative could be to develop an energy-efficient approach that formulates an auction to allow channel allocation in a CRN. We can think of reducing the communication cost and computational cost or managing the number of phases in the auction process to achieve an energy-efficient solution for the spectrum-allocation problem.

Author Contributions: Conceptualization, M.D., N.S., and S.K.D.; methodology, M.D., N.S., and S.K.D.; validation, M.D.; formal analysis, M.D. and N.S.; investigation, M.D. and S.K.D.; resources, M.D.; writing—original draft preparation, M.D.; writing—review and editing, M.D., N.S., and S.K.D.; supervision, N.S. and S.K.D. All authors have read and agreed to the published version of the manuscript.

Funding: This research received no external funding.

Conflicts of Interest: The authors declare that there is no conflict of interest.

\section{References}

1. Federal Communications Commission. FCC Document ET Docket No. Spectrum Policy Task Force Report; Federal Communications Commission: Washington, DC, USA, 2002; pp. 2-135.

2. Akyildiz, I.F.; Lee, W.-Y.; Vuran, M.C.; Mohanty, S. Next generation/dynamic spectrum access/cognitive radio wireless networks: A survey. Comput. Netw. 2006, 50, 2127-2159. [CrossRef]

3. Xing, X.; Jing, T.; Cheng, W.; Huo, Y.; Cheng, X. Spectrum prediction in cognitive radio networks. IEEE Wirel. Commun. 2013, 20, 90-96. [CrossRef]

4. Piran, M.J.; Pham, Q.-V.; Islam, S.M.R.; Cho, S.; Bae, B.; Suh, D.Y.; Han, Z. Multimedia communication over cognitive radio networks from QoS/QoE perspective: A comprehensive survey. J. Netw. Comput. Appl. 2020, 172, 1-44.

5. Song, M.; Xin, C.; Zhao, Y.; Cheng, X. Dynamic spectrum access: From cognitive radio to network radio. IEEE Wirel. Commun. 2012 19, 23-29. [CrossRef]

6. Ahmed, E.; Gani, A.; Abolfazli, S.; Yao, L.J.; Khan, S.U. Channel assignment algorithms in cognitive radio networks: Taxonomy, open issues, and challenges. IEEE Commun. Surv. Tutor. 2016, 18, 795-823. [CrossRef]

7. Tragos, E.Z.; Zeadally, S.; Fragkiadakis, A.G.; Siris, V.A. Spectrum assignment in cognitive radio networks: A comprehensive survey. IEEE Commun. Surv. Tutor. 2013, 15, 1108-1135. [CrossRef]

8. Parsons, S.; Rodriguez-Aguilar, J.A.; Klein, M. Auctions and bidding: A guide for computer scientists. ACM Comput. Surv. 2011, 43, 1-66. [CrossRef]

9. Zhang, Y.; Niyato, D.; Wang, P.; Hossain, E. Auction-based re-source allocation in cognitive radio systems. IEEE Commun. Mag. 2012, 50, 108-120. [CrossRef]

10. ITU. Assignment and Use of Radio Spectrum - Policy Guidelines and Economic Aspects; ITU: Geneva, Switzerland 2016.

11. $\mathrm{Hu}, \mathrm{F}$; Chen, B.; Zhu, K. Full spectrum sharing in cognitive radio networks toward 5G: A survey. IEEE Access 2018, 6, 15754-15776. [CrossRef]

12. Gupta, A.; Jha, R.K. A survey of 5g network: Architecture and emerging technologies. IEEE Access 2015, 3, 1206-1232. [CrossRef]

13. Khan, A.A.; Rehmani, M.H.; Rachedi, A. Cognitive-radio based internet of things: Applications, architectures, spectrum related functionalities, and future research directions. IEEE Wirel. Commun. 2017, 24, 17-25 [CrossRef]

14. Asif, A.R.; Zahra, F.; Matin, M.A. Cognitive solution for IoT communication technologies-Emphasis on 5G. J. Electr. Eng. 2020, 71, 131-137.

15. Devi, M.; Sarma, N.; Deka, S.K. Multi-winner heterogeneous spectrum auction mechanism for channel allocation in cognitive radio networks. In Proceedings of the 16th 2020 International Conference on Distributed Computing and Internet Technology (ICDCIT-2020), Bhubaneswar, India, 9-12 January 2020.

16. Akyildiz, I.F.; Lee, W.-Y.; Chowdhury, K.R. Crahns: Cognitive radio ad hoc networks. Ad Hoc Netw. 2009, 7, 810-836. [CrossRef]

17. Zheng, H.; Peng, C. Collaboration and fairness in opportunistic spectrum. In Proceedings of the 2005 IEEE International Conference on Communications (ICC), Seoul, Korea, 16-20 May 2005; pp. 3132-3136. 
18. Peng, C.; Zheng, H.; Zhao, B.Y. Utilization and fairness in spectrum assignment for opportunistic spectrum access. Mob. Netw. Appl. 2006, 11, 555-576. [CrossRef]

19. Niyato, D.; Hossain, E. Competitive pricing for spectrum sharing in cognitive radio networks: Dynamic game, inefficiency of nash equilibrium and collusion. IEEE J. Sel. Areas Commun. 2008, 26, 779-797. [CrossRef]

20. Zhao, Z.; Peng, Z.; Zheng, S.; Shang, J. Cognitive radio spectrum allocation using evolutionary algorithms. IEEE Trans. Wirel. Commun. 2009, 8, 4421-4425. [CrossRef]

21. Wang, X.; Li, Z.; Xu, P.; Xu, Y.; Gao, X.; Chen, H.-H. Spectrum sharing in cognitive radio networks-an auction based approach. IEEE Trans. Syst. Man Cybern. 2010, 40, 587-596. [CrossRef]

22. Kash, I.A.; Murthy, R.; Parkes, D.C. Enabling spectrum sharing in secondary market auctions. IEEE Trans. Mob. Comput. 2014, 3, 556-568. [CrossRef]

23. Khaledi, M.; Abouzeid, A.A. Auction-based spectrum sharing in cognitive radio networks with heterogeneous channels. In Proceedings of the Information Theory and Applications Workshop (ITA), San Diego, CA, USA, 10-15 February 2013.

24. Amraoui, A.; Benmammar, B.; Krief, F.; Bendimerad, F.T. Auction-based agent negotiation in cognitive radio ad hoc network. In Proceedings of the 2012 International Conference on Ad Hoc Networks, Paris, France, 16-17 October 2012; pp. 119-134.

25. Hyder, C.S.; Jeitschko, T.D.; Xiao, L. Bid and time truthful on-line auctions in dynamic spectrum markets. IEEE Trans. Cogn. Commun. Netw. 2017, 3, 82-96. [CrossRef]

26. Gao, L.; Wang, X.; Xu, Y.; Zhang, Q. Spectrum trading in cognitive radio networks : A contract-theoretic modeling approach. IEEE J. Sel. Areas Commun. 2011, 29, 843-855. [CrossRef]

27. Shi, Z.; Luo, G. Multi-band spectrum allocation algorithm based on first-price sealed auction. Cybern. Inf. Technol. 2017, 17, 104-112. [CrossRef]

28. Bae, J.; Beigman, E.; Berry, R.A.; Honig, M.L.; Vohra, R. Sequential bandwidth and power auctions for distributed spectrum sharing. IEEE J. Sel. Areas Commun. 2008, 26, 1193-1203. [CrossRef]

29. Jia, J.; Zhang, Q.; Zhang, Q.; Liu, M. Revenue generation for truthful spectrum auction in dynamic spectrum access. In Proceedings of the 2009 International Symposium on Mobile Ad hoc Networking and Computing (MobiHoc), New Orleans, LA, USA, 18-21 May 2009; pp. 3-12.

30. Devi, M.; Sarma, N.; Deka, S.K.; Chauhan, P. Sequential bidding auction mechanism for spectrum sharing in cognitive radio networks. In Proceedings of the 2017 International Conference on Advanced Net-Works and Telecommunications Systems (ANTS), Bhubaneswar, India, 17-20 December 2017.

31. Zhou, X.; Gandhi, S.; Suri, S.; Zheng, H. eBay in the sky: Strategy-proof wireless spectrum auctions. In Proceedings of the 14th 2008 International Conference on Mobile Computing and Networking (MobiCom), San Francisco, CA, USA, 13-19 September 2008; pp. 2-13.

32. Zhai, X.; Zhou, T.; Zhu, C.; Chen, B.; Fang, W.; Zhu, K. Truthful double auction for joint internet of energy and profit optimization in cognitive radio networks. IEEE Access 2018, 6, 23180-23190. [CrossRef]

33. Khairullah, E.F.; Chatterjee, M. PreDA: Preference-based double auction for spectrum allocation in heterogeneous DSA networks. Comput. Commun. 2019, 133, 41-50. [CrossRef]

34. Dong, W.; Rallapalli, S.; Qiu, L.; Ramakrishnan, K.K.; Zhang, Y. Double auctions for dynamic spectrum allocation. IEEE/ACM Trans. Netw. 2016, 24, 2485-2497. [CrossRef]

35. Zhang, X.; Yang, D.; Xue, G.; Yu, R.; Tang, J. Transmitting and sharing: A truthful double auction for cognitive radio networks. In Proceedings of the 2018 IEEE ICC, Kansas City, MO, USA, 20-24 May 2018.

36. Chen, Y.; Zhang, J.; Wu, K.; Zhang, Q. TAMES: A truthful double auction for multi-demand heterogeneous spectrums. IEEE Trans. Parallel Distrib. Syst. 2014, 25, 3012-3024. [CrossRef]

37. Chen, Y.; Lin, P.; Zhang, Q. Lotus: Location-aware online truthful double auction for dynamic spectrum access. IEEE Trans. Wirel. Commun. 2015, 1092-1099. [CrossRef]

38. Shafiq, M.; Choi, J.-G. Adaptive auction framework for spectrum market in cognitive radio networks. J. Netw. Syst. Manag. 2017, 26, 518-546. [CrossRef]

39. Wang, X.; Huang, L.; Xu, H.; Huang, H. Auction-based resource allocation for cooperative cognitive radio networks. Comput. Commun. 2017, 47, 40-51. [CrossRef]

40. Zhang, X.; Zhang, B.; An, K.; Chen, Z.; Guo, D. Auction-based secondary relay selection on overlay spectrum sharing in hybrid satellite-terrestrial sensor networks. Sensors 2019, 19, 5039. [CrossRef]

41. Brandon, F.L. A survey of common control channel design in cognitive radio networks. Phys. Commun. 2011, 4, $26-39$.

42. Xiang, J.; Zhang, Y.; Skeie, T. Medium access control protocols in cognitive radio networks. Wirel. Commun. Mob. Comput. 2009, 10, 31-49. [CrossRef]

43. Kim, H.; Shin, K.G. Efficient discovery of spectrum opportunities with mac-layer sensing in cognitive radio networks. IEEE Trans. Mob. Comput. 2008, 7, 533-545.

44. Chen, Z.; Huang, H.; Huang, L. True-MCSA: A framework for truthful double multi-channel spectrum auctions. IEEE Trans. Wirel. Commun. 2013, 12, 3838-3850. [CrossRef] 
45. Gupta, S.; Malagar, V. IEEE 802.22 Standard for Regional Area Networks. In Proceedings of the 2017 International Conference on Next Generation Computing and Information Systems (ICNGCIS), Jammu, India, 19-21 July 2017.

46. Flores, A.B.; Guerra, R.E.; Knightly, E.W.; Ecclesine, P.; Pandey, S. IEEE 802.11af: A standard for TV white space spectrum sharing. IEEE Commun. Mag. 2013, 51, 92-100. [CrossRef] 